\title{
A Monte Carlo Study of the Momentum Dependence on the Results of Tracking Unknown Particle Species in the BaBar Detector
}

\author{
Stephen Sewerynek
}

SLAC-R-838

\author{
Prepared for the Department of Energy \\ under contract number DE-AC02-76SF00515
}

Printed in the United States of America. Available from the National Technical Information Service, U.S. Department of Commerce, 5285 Port Royal Road, Springfield, VA 22161. 
This document, and the material and data contained therein, was developed under sponsorship of the United States Government. Neither the United States nor the Department of Energy, nor the Leland Stanford Junior University, nor their employees, nor their respective contractors, subcontractors, or their employees, makes an warranty, express or implied, or assumes any liability of responsibility for accuracy, completeness or usefulness of any information, apparatus, product or process disclosed, or represents that its use will not infringe privately owned rights. Mention of any product, its manufacturer, or suppliers shall not, nor is it intended to, imply approval, disapproval, or fitness of any particular use. A royalty-free, nonexclusive right to use and disseminate same of whatsoever, is expressly reserved to the United States and the University. 


\title{
A MONTE CARLO STUDY OF THE MOMENTUM DEPENDENCE ON THE RESULTS OF TRACKING UNKNOWN PARTICLE SPECIES IN THE BABAR DETECTOR
}

\author{
By \\ Stephen Sewerynek \\ B.Sc.(Hon), University of Toronto, 1996 \\ A THESIS SUBMITTED IN PARTIAL FULFILLMENT OF \\ THE REQUIREMENTS FOR THE DEGREE OF \\ Master of SCIEnCE \\ in \\ THE FACULTY OF GRADUATE STUDIES \\ DEPARTMENT OF PHYSICS AND ASTRONOMY
}

We accept this thesis as conforming

to the required standard

THE UNIVERSITY OF BRITISH COLUMBIA

April 1999

(C) Stephen Sewerynek, 1999 
In presenting this thesis in partial fulfillment of the requirements for an advanced degree at the University of British Columbia, I agree that the Library shall make it freely available for reference and study. I further agree that permission for extensive copying of this thesis for scholarly purposes may be granted by the head of my department or by his or her representatives. It is understood that copying or publication of this thesis for financial gain shall not be allowed without my written permission.

Department of Physics and Astronomy

The University of British Columbia

6224 Agricultural Road

Vancouver, BC, Canada

V6T 1Z1

Date: 


\section{Abstract}

The BABAR experiment is composed of an international collaboration that will test the Standard Model prediction of $C P$ violation. To accomplish this a new detector was constructed at the asymmetric $B$ Factory, located at the Stanford Linear Accelerator Center. The tests will shed some light on the origins of $C P$ violation, which is an important aspect in explaining the matter/antimatter asymmetry in the universe. In particular, the $\mathrm{BABAR}$ experiment will measure $C P$ violation in the neutral $B$ meson system.

In order to succeed, the BABAR experiment requires excellent track fitting and particle species identification. Prior to the current study, track fitting was done using only one particle species - the pion. But given the momentum dependence on the accuracy of the results from this choice of particle species, a better algorithm needed to be developed. Monte Carlo simulations were carried out and a new algorithm utilizing all five particle species present in the BABAR detector was created. 


\section{Table of Contents}

$\begin{array}{ll}\text { Abstract } & \text { ii }\end{array}$

List of Tables $\quad$ vi

List of Figures $\quad$ vii

Quotes $\quad$ ix

$\begin{array}{ll}\text { Acknowledgments } & \mathrm{x}\end{array}$

1 Introduction $\quad 1$

1.1 The Sakharov Criteria ........................ 1

1.1.1 Baryon Non-Conservation .............. 2

1.1.2 The Process of $C P$ Violation . . . . . . . . . . 2

1.1.3 Thermal Equilibrium Lost .............. 2

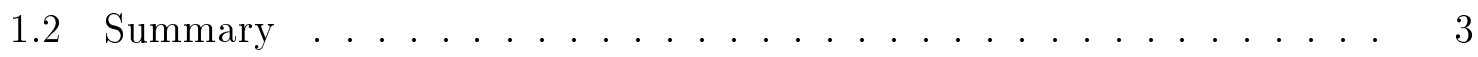

$2 C P$ Violation $\quad 4$

2.1 A Brief History of $C P$ Violation . . . . . . . . . . . . 5

2.1.1 The Neutral Kaon System . . . . . . . . . . . . 5

2.2 The Neutral B Mesons . . . . . . . . . . . . . . . . . 9 9

2.3 Three Types of $C P$ Violation in $B$ Decays $\ldots \ldots \ldots$

2.3.1 CP Violation in Decay . . . . . . . . . . . . 11 
$2.3 .2 \quad C P$ Violation in Mixing . . . . . . . . . . . . 11

2.3.3 CP Violation in the Interference Between Decays With and Without Mixing . . . . . . . . . . . . . . 12

$2.4 C P$ Violation in BABAR . . . . . . . . . . . . . . . . 12

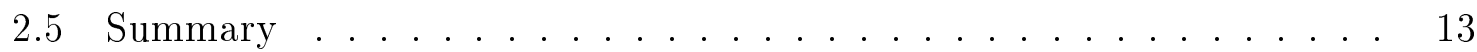

3 The BaBar Detector $\quad 14$

3.1 The Silicon Vertex Tracker . . . . . . . . . . . . . . . . 16

3.2 The Drift Chamber . . . . . . . . . . . . . . . . . . . 19

3.2.1 The Drift Chamber Design . . . . . . . . . . . . . . . 19

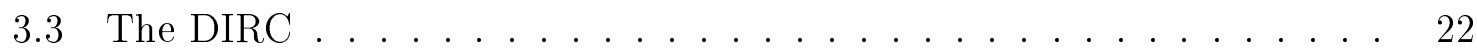

3.4 The Electromagnetic Calorimeter . . . . . . . . . . . . . 24

3.5 The Instrumented Flux Return _ . . . . . . . . . . . . 25

3.6 Summary . . . . . . . . . . . . . . . . . . 27

4 The Drift Chamber 28

4.1 Ionization Detectors $\ldots \ldots \ldots \ldots \ldots \ldots$

4.2 Gas Properties . . . . . . . . . . . . . . . . . . 30

4.3 Gas Type Choice . . . . . . . . . . . . . . . . . . . . 31

4.3 .1 Electron Diffusion . . . . . . . . . . . . . . . . 32

4.3 .2 Drift Velocity . . . . . . . . . . . . . . . . 34

4.4 A Drift Chamber in a Magnetic Field . . . . . . . . . . . . . . 34

4.5 Summary . . . . . . . . . . . . . . . . . 36

5 The Silicon Vertex Tracker $\quad 37$

5.1 Basic Semiconductor Properties . . . . . . . . . . . . . 37

5.1 .1 Charge Carriers . . . . . . . . . . . . . . . 38 
5.1.2 Intrinsic Charge Carrier Concentration . . . . . . . . . . 39

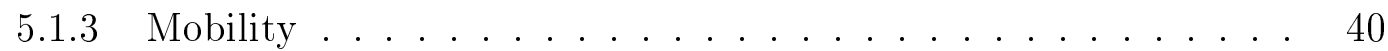

5.2 The np Semiconductor Junction . . . . . . . . . . . . 41

5.3 Micro-Strip Detectors . . . . . . . . . . . . 42

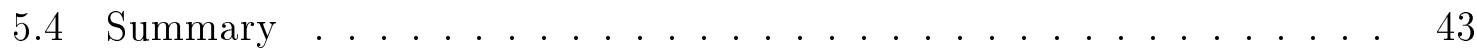

6 Tracking Reconstruction $\quad 44$

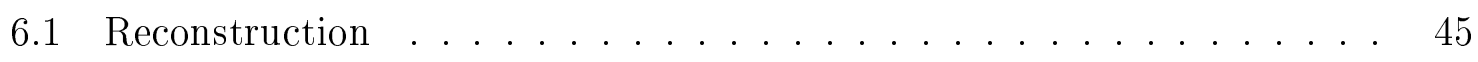

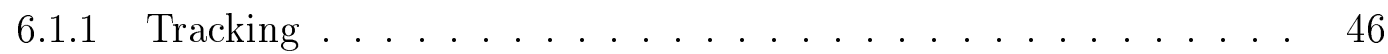

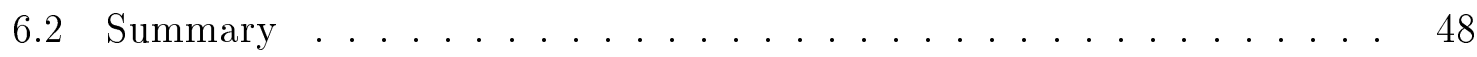

7 Multiple Mass Hypothesis Analysis $\quad 49$

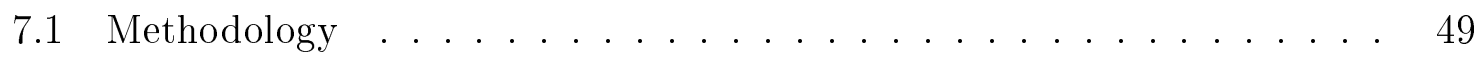

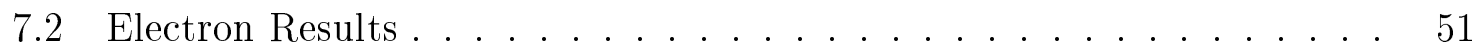

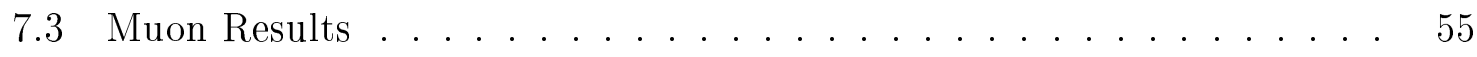

7.4 Kaon Results ......................... 59

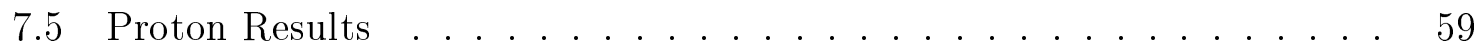

7.6 The Effect of Changing the Sigma Value . . . . . . . . . . . 59

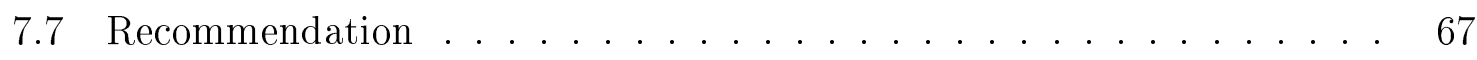

$\begin{array}{lll}8 & \text { Conclusion } & 69\end{array}$

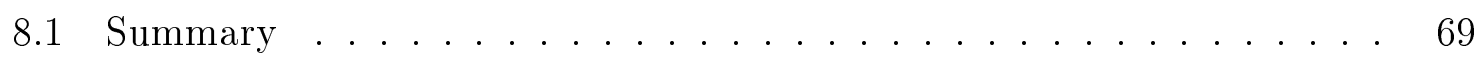

$\begin{array}{ll}\text { Bibliography } & 70\end{array}$ 


\section{List of Tables}

7.1 Electron Momentum Cutoffs $(\mathrm{GeV} / \mathrm{c})$ for $\Delta S<0.1 \sigma$ at a $95 \% \mathrm{CL} \ldots . \quad 55$

7.2 Muon Momentum Cutoffs $(\mathrm{GeV} / \mathrm{c})$ for $\Delta S<0.1 \sigma$ at a $95 \% \mathrm{CL} \ldots . \quad 55$

7.3 Kaon Momentum Cutoffs $(\mathrm{GeV} / \mathrm{c})$ for $\Delta S<0.1 \sigma$ at a $95 \% \mathrm{CL} \ldots \ldots 59$

7.4 Proton Momentum Cutoffs $(\mathrm{GeV} / \mathrm{c})$ for $\Delta S<0.1 \sigma$ at a $95 \% \mathrm{CL} \ldots .66$

7.5 Momentum Cutoffs $(\mathrm{GeV} / \mathrm{c})$ for Various Values of $\Delta S$ at a $95 \% \mathrm{CL} \ldots 67$ 


\section{List of Figures}

2.1 Cartoon of an $e^{+} e^{-}$collision and the resulting $B \bar{B}$ pair. . . . . . . . 9

3.1 Layout of the BABAR detector . . . . . . . . . . . . 15

3.2 SVT view in a plane containing the beam axis. . . . . . . . . . 17

3.3 Cross-sectional view of the SVT in the plane orthogonal to the beam axis. 18

3.4 Side view of the BABAR drift chamber. . . . . . . . . . . . . 20

3.5 Cell layout in the BABAR drift chamber. . . . . . . . . . . . . 21

3.6 Schematic of a single radiator bar of a DIRC counter. . . . . . . . . 23

3.7 Side view of the EMC calorimeter. . . . . . . . . . . . . 24

3.8 The IFR Barrel . . . . . . . . . . . . . . . . . . . 26

4.1 Side view of the BABAR drift chamber. . . . . . . . . . . . . 29

$4.250 \mathrm{~ns}$ isochrones in a typical BABAR drift chamber cell. . . . . . . . 35

5.1 Energy band structure of insulators, semiconductors, and metals. . . . 38

5.2 Schematic diagram of an np junction. . . . . . . . . . . 42

$7.1 \Delta S$ vs. Monte Carlo Momentum for $p_{z}, d, \phi$, and $z$ for an Electron. . . 52

$7.2 \Delta S\left(p_{t}\right)$ vs. Monte Carlo Momentum for an Electron. . . . . . . . . 53

7.3 Electron $\Delta S\left(p_{t}\right)$ distribution for $2.1 \leq p \leq 2.2 \mathrm{GeV} / \mathrm{c} \ldots \ldots$. . . . 54

$7.4 \Delta S$ vs. Monte Carlo Momentum for $p_{z}, d, \phi$, and $z$ for a Muon. . . . 56

$7.5 \Delta S\left(p_{t}\right)$ vs. Monte Carlo Momentum for a Muon. . . . . . . . . . 57

7.6 Muon $\Delta S\left(p_{t}\right)$ distribution for $0.5 \leq p \leq 0.6 \mathrm{GeV} / \mathrm{c} \ldots \ldots$ 
$7.7 \Delta S$ vs. Monte Carlo Momentum for $p_{t}, d, \phi$, and $z$ for a Kaon. . . . 60

$7.8 \Delta S\left(p_{z}\right)$ vs. Monte Carlo Momentum for a Kaon . . . . . . . . . 61

7.9 Kaon $\Delta S\left(p_{z}\right)$ distribution for $2.0 \leq p \leq 2.1 \mathrm{GeV} / \mathrm{c} \ldots \ldots 2$

$7.10 \Delta S$ vs. Monte Carlo Momentum for $p_{z}, d, \phi$, and $z$ for a Proton . . . 63

$7.11 \Delta S\left(p_{t}\right)$ vs. Monte Carlo Momentum for a Proton . . . . . . . . . 64

7.12 Proton $\Delta S\left(p_{t}\right)$ distribution for $3.1 \leq p \leq 3.2 \mathrm{GeV} / \mathrm{c} \ldots \ldots$

7.13 The Effect of Changing the Sigma Value on Cutoff Momentum. . . . . 66 


\section{Quotes}

In the beginning the Universe was created. This has made a lot of people very angry and has been widely regarded as a bad move.

- Douglas Adams

I don't pretend to understand the Universe - it's a great deal bigger than I am.

- Thomas Carlyle

A friend of mine once sent me a post card with a picture of the entire planet Earth taken from space. On the back it said, "Wish you were here."

- Steven Wright 


\section{Acknowledgments}

Let's see whom do I have to thank? Ah yes, I suppose that I owe the most gratitude to my supervisor Dr. Janis McKenna. Her energy rivals that of the Energizer bunny and without it I fear that she would not have been able to correct my many grammar and typing mistakes. I especially wish to thank her for all those free dinners that she and her husband paid for - 3 of which were steak.

My thanks also go out to Chris Hearty and Dave Axen for providing comments and corrections.

My fellow office dwellers also deserve some credit for putting up with my choice of music and sometimes spontaneous outbursts. They include Douglas Thiessen, Jiansen Lu, and Roby Austin.

While at SLAC I got to enjoy the many benefits of being around so many intelligent people at the top of their field - I also got the enjoy the thrill of taking unsanctioned electric cart rides down the klystron gallery and down to the BABAR pit area. I would like to thank Stephen Schaffner for putting up with my endless amount of questions and Charlie Young for dealing with all my Beta concerns.

Lastly I would like to thank my parents for always putting up with my sometimes crazy ideas and their continued support of all my endeavours. The fact that they are half way across the country has reduced my stress level by many orders of magnitude and 
I am sure that after reading this thesis they will come to the conclusion that their son is further out there than mere geography dictates. 


\section{Chapter 1}

\section{Introduction}

The Big Bang created the universe some 14 billion year ago. The very early universe was filled with matter, antimatter, and energy. Two of these quantites, matter and energy, are still prevalent in today's universe. But one may then ask, "what happened to all the antimatter?" Upon combination, matter and antimatter annihilate one another in a burst of energy. If the early universe consisted of equal amounts matter and antimatter then we would not exist today. One is then forced to suppose that there was a slight asymmetry between the quantities of matter/antimatter which would then account for there being any matter in the present day universe at all.

\subsection{The Sakharov Criteria}

In an attempt to explain the matter/antimatter asymmetry in the universe A.D. Sakharov [1] proposed that the following three requirements be satisfied: (i) a nonconservation of baryons, (ii) a process of $C P$ violation, and (iii) the absence of thermodynamic equilibrium. 


\subsubsection{Baryon Non-Conservation}

The asymmetry between matter/antimatter manifests itself in an imbalance between the numbers of baryons and antibaryons present in the universe. If such a baryon/antibaryon imbalance does exist it would mean that baryons could decay into non-baryons (the opposite being true as well). As an example, imagine a baryon, $X$, decaying into quarks, $q$, and an electron, $e^{-}$, through the reaction $X \rightarrow q \bar{q}+e^{-}$(similarly an antibaryon through the process $\bar{X} \rightarrow \bar{q} q+e^{+}$). If this process were possible, then baryon number would not be conserved as the products on the right side of the above equations are mesons and leptons. Although certain Grand Unified Theories (GUTs) predict baryon non-conservation, to date no experimental evidence has been gathered to support the existence of baryon number non-conservation.

\subsubsection{The Process of $C P$ Violation}

Taken alone, a baryon non-conserving interaction could not likely account for all the matter/antimatter irregularity in the universe. The only way an excess of quarks over antiquarks could develop is if the partial decay rate of the $X$ to a quark was slightly greater than that of an $\bar{X}$ to an antiquark. This mechanism, of favouring the production of matter over antimatter (or vice-versa), is called the process of $C P$ violation. This important subject is dealt with in more detail in Chapter 2.

\subsubsection{Thermal Equilibrium Lost}

The last Sakharov condition deals with the evolution of the universe from a state of thermal equilibrium to a state of non-equilibrium. This is needed because while the universe is in thermal equilibrium matter and antimatter are constantly being interchanged with energy. In such a state, any asymmetry between the matter and 
antimatter that may have developed would be erased.

The current scenario for the evolution of the universe is that initially baryons and antibaryons existed in equal proportions. At some later time, processes that violate $C P$ and baryon number conservation started to occur which led to an excess of baryons over antibaryons. This imbalance was preserved by having the universe out of thermal equilibrium. After a short while the baryon number violating reactions were suppressed and the baryon excess in the universe was "frozen in." The remaining antibaryons combined with some of the baryons and produced photons. After this, the only particles left over were photons and baryons, which is what populates the universe today.

\section{$1.2 \quad$ Summary}

As the previous argument outlined, $C P$ violation is an essential ingredient in the recipe of matter/antimatter asymmetry. The BABAR experiment will experimentally examine this important aspect in explaining the evolution of the universe.

Excellent track fitting and particle species identification is essential for the BABAR experiment to succeed. This thesis will deal with the investigation of multiple fittings of tracks of unknown species in the BABAR detector. Of the five types of charged particle species detected (electrons, muons, pions, kaons, and protons), the most abundant are the pions. The identification of these tracks are not known a priori. Because of this, all tracks are tracked as a pion by default. Given the momentum dependence on the accuracy of results from this default choice of particle species, a better algorithm needed to be developed. Specifically, at what momentum is it beneficial, in terms of accuracy, to track the unknown particles as all five particle species rather than as just the default pion choice? 


\section{Chapter 2}

\section{$C P$ Violation}

There are three discrete transformations one can perform on a given object that are of interest in the attempt to understand $C P$ violation. The first is charge conjugation, denoted by $C$. When performed it changes the particle into its antiparticle. Consider a state $X \mid \vec{x}, t>$, when acted upon by $C$ :

$$
C X|\vec{x}, t>=\bar{X}| \vec{x}, t>
$$

where $\bar{X}$ denotes the antiparticle of X. Another transformation, parity or $P$, replaces an object with its mirror image and rotates it 180 degrees about the axis perpendicular to the mirror. Using the same state as above one gets,

$$
P X|\vec{x}, t>=X|-\vec{x}, t>
$$

Another operation is called time reversal or just simply $T$. As its name implies it reverses the direction of time when applied to a state

$$
T X|\vec{x}, t>=X| \vec{x},-t>
$$

A system is said to exhibit a given symmetry if the action of a certain transformation leaves that system unchanged as far as physical laws are concerned. Charge conjugation and parity when applied individually are not symmetries of nature. It was thought that 
the combination of $C P$ would however be a conserved quantity. This idea was shattered in the early 1960's when it was discovered that the kaons, $K_{S}^{0}$ and $K_{L}^{0}$, are not invariant with respect to $C P$ symmetry [2]. Later it was shown that the Standard Model (SM) does indeed allow $C P$ to be violated in the presence of three quark generations [3]. However the allowed amount of $C P$ violation in the SM is likely not sufficient to account for the level of asymmetry needed to explain the matter/antimatter asymmetry of the universe [4].

Below is a further explanation of what $C P$ violation is and how its measured. For an excellent and more detailed review and discussion of $C P$ violation please see section 1 in The BABAR Physics Book [5].

\subsection{A Brief History of $C P$ Violation}

It is beneficial first to see where one has been before attempting to go further. $C P$ violation was first discovered in the neutral kaon system and it is with this topic that this discussion begins.

\subsubsection{The Neutral Kaon System}

Kaons were discovered in cosmic rays by physicists in Europe shortly after the end of the Second World War. They are neutral particles that decay into positive and negative (or neutral) pions. Because of their relatively long life-times they were assigned a quantum number called strangeness. The $K^{0}$ meson is assigned a strangeness of +1 and the pions it decays to are each said to have 0 strangeness. The antiparticle partner

to the neutral kaon, the $\bar{K}^{0}$, is given a strangeness of -1 . The $K^{0}$ and $\bar{K}^{0}$ both decay 
into two pions

$$
\begin{aligned}
& K^{0} \rightarrow \pi^{+} \pi^{-}, K^{0} \rightarrow \pi^{0} \pi^{0} \\
& \bar{K}^{0} \rightarrow \pi^{+} \pi^{-}, \bar{K}^{0} \rightarrow \pi^{0} \pi^{0}
\end{aligned}
$$

It was unusual to have separate particles decaying into exactly the same final state. So much so that there was a real problem of how you should distinguish them at all. In 1955 Murray Gell-Mann and Abraham Païs postulated that the $K^{0}$ and $\bar{K}^{0}$ are coupled via their final states and that during decays are not observed separately but in two "normal modes" characterized by two different lifetimes [6]. Gell-Mann and Païs said that $C P$ symmetry would reveal information about the "normal modes" or the proper linear combination of $K^{0}$ and $\bar{K}^{0}$. Letting the $C P$ operation act on a state of two pions one gets

$$
\begin{aligned}
C P \mid \pi^{+} \pi^{-}> & =(-1)^{2} \mid \pi^{+} \pi^{-}> \\
& =+1 \mid \pi^{+} \pi^{-}>
\end{aligned}
$$

since pions are odd under a parity transformation. Now if one tries to perform the same operation on a system of three pions the outcome turns out to be a state that has a $C P$ eigenvalue of -1 .

$$
\begin{aligned}
C P \mid \pi^{+} \pi^{-} \pi^{0}> & =(-1)^{3} \mid \pi^{+} \pi^{-} \pi^{0}> \\
& =-1 \mid \pi^{+} \pi^{-} \pi^{0}>
\end{aligned}
$$

$C P$ acting on any state twice gives back the initial state again,

$$
(C P)^{2} X|\vec{x}, t>=X| \vec{x}, t>.
$$

This characteristic of the $C P$ operation can be summed up by saying that it has two eigenvalues, +1 and -1 . This lead to a definition for the two "normal modes" of the $K$ 
system; one combination being symmetric and the other antisymmetric:

$$
\begin{aligned}
& \mid K_{1}>=\frac{1}{\sqrt{2}}\left(\left|K^{0}>+\right| \bar{K}^{0}>\right) \\
& \mid K_{2}>=\frac{1}{\sqrt{2}}\left(\left|K^{0}>-\right| \bar{K}^{0}>\right) .
\end{aligned}
$$

Solving for $K^{0}$ and $\bar{K}^{0}$ one finds

$$
\begin{aligned}
\mid K^{0}> & =\frac{1}{\sqrt{2}}\left(\left|K_{1}>+\right| K_{2}>\right) \\
\mid \bar{K}^{0}> & =\frac{1}{\sqrt{2}}\left(\left|K_{1}>-\right| K_{2}>\right) .
\end{aligned}
$$

Described in this way, if $C P$ were a perfect symmetry, a beam of $K^{0}$ mesons would be composed of equal parts $K_{1}$ and $K_{2}$. The action of $C P$ on $\mid K^{0}>$ and $\mid \bar{K}^{0}>$ may be deduced from the above definitions:

$$
\begin{aligned}
& C P\left|K^{0}>=\right| \bar{K}^{0}> \\
& C P\left|\bar{K}^{0}>=\right| K^{0}>
\end{aligned}
$$

If $C P$ was respected by the mechanism responsible for the decay of the $K$ meson system then the final state would either consist of two or three pions. Letting $C P$ act on the symmetric state, $K_{1}$, gives back the same state with an eigenvalue of +1 .

$$
\begin{aligned}
C P \mid K_{1}> & =C P\left(\frac{1}{\sqrt{2}}\right)\left(\left|K^{0}>+\right| \bar{K}^{0}>\right) \\
& =\frac{1}{\sqrt{2}}\left(\left|\bar{K}^{0}>+\right| K^{0}>\right) \\
& =\mid K_{1}>
\end{aligned}
$$

But $C P$ acting on the antisymmetric state, $K_{2}$, yields an eigenvalue of -1 .

$$
C P \mid K_{2}>=C P\left(\frac{1}{\sqrt{2}}\right)\left(\left|K^{0}>-\right| \bar{K}^{0}>\right)
$$




$$
\begin{aligned}
& =\frac{1}{\sqrt{2}}\left(\left|\bar{K}^{0}>-\right| K^{0}>\right) \\
& =-1\left(\frac{1}{\sqrt{2}}\right)\left(\left|K^{0}>-\right| \bar{K}^{0}>\right) \\
& =-1 \mid K_{2}>
\end{aligned}
$$

It was found that the $K_{1}$ had a shorter lifetime than the $K_{2}$, which lead to the convention of calling the symmetric state $K_{S}$ and the antisymmetric state $K_{L}$. So if $C P$ was conserved in the $K$ system then the symmetric state could only decay into two pions (but not three) and the antisymmetric state is forced to decay into three pions only (not two). Since the rate of decay for a particle system depends on the excess energy available (or phase space), the $K_{S}$ and $K_{L}$ will have dramatically different lifetimes because the mass of a $K$ is $\sim 500 \mathrm{MeV}$ while the mass of a pion is only $\sim 140 \mathrm{MeV}$. It is clear to see that there is more kinetic energy available, hence more phase space, for the two pion decay than the three pion decay. This huge difference in phase space is why there is such a large difference in the $K_{S}^{0}$ and $K_{L}^{0}$ lifetimes. The lifetime of a $K_{S}$ is only $0.8927 \times 10^{-10} \mathrm{~S}$, compared to the lifetime of a $K_{L}$ which is $5.17 \times 10^{-8} \mathrm{~S}$ [7]. A beam of kaons would initially contain equal parts of $K_{S}$ and $K_{L}$ but if one waited long enough, all the $K_{S}$ would decay and only the $K_{L}$ would remain. This fact also provided a test of $C P$ symmetry. Since the $K_{L}$ is odd under $C P$ then it is forbidden to decay into two pions, $\pi^{+} \pi^{-}$, a $C P$ even state. It was experimentally discovered that $K_{L}$ did indeed decay into $\pi^{+} \pi^{-}$about 1 out of every 500 times [8]. It then had to be conceded that $C P$ was not a perfect symmetry after all, but just a nearly perfect symmetry. 


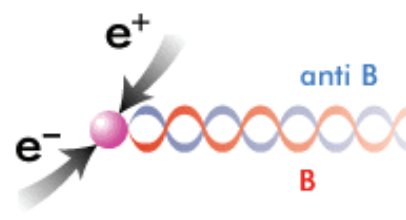

Figure 2.1: Cartoon of an $e^{+} e^{-}$collision and the resulting $B \bar{B}$ pair.

\subsection{The Neutral B Mesons}

A neutral $B_{d}$ meson is composed of two quarks, a $b$ type quark or antiquark and one $d$ type. It is sometimes denoted as $B_{d}$ but in the following it will be abbreviated as simply $B^{0}$. Similar to the kaons discussed above, the physical neutral $B^{0}$ mesons $\left(B_{H}^{0}\right.$ and $\left.B_{L}^{0}\right)$ are also linear combinations of $B^{0}$ and $\bar{B}^{0}$. Instead of a long-lived and short-lived combination the $B^{0}$ mesons are combined such that

$$
\begin{aligned}
& \left|B_{L}>=p\right| B^{0}>+q \mid \bar{B}^{0}> \\
& \left|B_{H}>=p\right| B^{0}>-q \mid \bar{B}^{0}>
\end{aligned}
$$

where the subscripts refer to light and heavy mass eigenstates respectively (In the kaon system the lifetimes differ vastly, yet the masses are very close, while in the $B$ system, the masses are expected to differ significantly more than their lifetimes). The time evolution mixes the two $B^{0}$ and $\vec{B}^{0}$ states as one changes into the other (and back again - equation 2.17).

$$
B^{0} \rightarrow \bar{B}^{0} \rightarrow B^{0}
$$

The $B^{0}$ and $\bar{B}^{0}$ mesons produced at SLAC are in a coherent $L=1$ state. The total 
angular momentum for a system is given by

$$
J=L+S
$$

where $L$ is the angular momentum and $S$ is the combined spin of the particles. SLAC is operating at the $\Upsilon(4 S)$ resonance which is a $J=1$ state. Since the $B^{0}$ and $\bar{B}^{0}$ produced from the decay of the $\Upsilon(4 S)$ particle have zero spin they must be in an $L=1$ state for the conservation of angular momentum (equation 2.18) to hold. All this means is that the two components of a physical $B$ meson evolve in a coherent state so that at anytime there is exactly one $B^{0}$ and one $\bar{B}^{0}$ present (figure 2.1), as Bose symmetry states that a pair of identical bosons would have to be in a symmetric state. If two $B$ 's are identical then the overall wavefunction would be symmetric, but the $L=1$ state is antisymmetric. Therefore, the two components of a $B$ meson cannot both be a $B^{0}$ or $\bar{B}^{0}$, but must be one of each. When one of the $B^{0}$ particles decays, the other continues to evolve, and depending on the time difference between the decays of the first and second $B^{0}$, there is a possibility of detecting an event with two final state $B^{0}$ s or $\bar{B}^{0}$ s. To measure $C P$ asymmetries one looks for events were one $B$ decays to a final $C P$ eigenstate and the other to a "tagging" mode - that is one that identifies its b-flavour.

\subsection{Three Types of $C P$ Violation in $B$ Decays}

In the Standard Model the $B$ mesons can violate $C P$ symmetry in one of the following three ways: (i) $C P$ violation in decay, (ii) direct $C P$ violation in mixing, and (iii) $C P$ violation in the interference between decays with and without mixing. To date there is no experimental evidence for $C P$ violation in $B$ decays at all. 


\subsection{1 $C P$ Violation in Decay}

This occurs when the transitions to the final states for the $B^{0}$ and $\bar{B}^{0}$ are not of equal magnitude. As an example, if a $B^{0}$ decays according to,

$$
B^{0} \rightarrow X
$$

where $X$ is a final state consisting of 2 or more particles, then the corresponding $\bar{B}^{0}$ decay would look like

$$
\bar{B}^{0} \rightarrow \bar{X}
$$

where $\bar{X}$ is the charge conjugate of the $X$ state. If the decay rate for the processes in equations 2.19 and 2.20 are not equal then the system exhibits $C P$ violation in decay. This is often referred to as direct $C P$ violation. The asymmetries in charged $B$ decays are from $C P$ violation in decay.

\subsection{2 $C P$ Violation in Mixing}

This occurs when the mass eigenstates are different from the weak eigenstates. This type of $C P$ violation has been seen in the neutral kaon system. It is also referred to as indirect $C P$ violation. The magnitude of this effect would be dependent on the difference in lifetimes of the two $B$ decays. Since the neutral $B$ mesons have a negligible fractional difference in lifetime, $\mathcal{O}\left(10^{-2}\right)$, this effect is expected to be small in the $B$ system, but is large in the kaon system, where the $K$ masses differ by 2 orders of magnitude. 


\subsection{3 $C P$ Violation in the Interference Between Decays With and Without Mixing}

To explore this effect consider a neutral $B$ decay into a final $C P$ eigenstate. Both the $B^{0}$ and $\bar{B}^{0}$ can decay to the same final state. To observe the $C P$ violation, the decays of a time-evolving neutral $B^{0}$ state that began as a $B^{0}$ at time zero are compared to one that started out as a $\bar{B}^{0}$. If one starts with a $B^{0}$, then the amplitude for the process

$$
B^{0} \rightarrow \bar{B}^{0} \rightarrow X
$$

interferes with the process in equation 2.19. The quantum mechanical interference between these two processes leads to $C P$ violation in the system.

\section{$2.4 C P$ Violation in $\mathbf{B}_{\mathrm{A}} \mathbf{B}_{\mathrm{AR}}$}

The BABAR experiment is designed to be very sensitive to the $C P$ violation denoted as the third type described above (although BABAR is also sensitive to the first two types - it is optimized for the third). Experiments done at SLAC in 1983 found that the $B$ mesons have a lifetime of order of a picosecond. This long lifetime is beneficial, as it improves the chances that a $B^{0}$ would oscillate into a $\bar{B}^{0}$ before decaying. Because the lifetime is of the order of the $B^{0}$ mixing parameter, one would expect $\sim 1 B^{0} \rightarrow \bar{B}^{0}$ oscillation before the $B$ decayed. $B$ mesons can be easily produced from an electron positron collider tuned to a center of mass energy of $\sim 10 \mathrm{GeV}$, which is the $\Upsilon(4 S)$ resonance. If the beams have equal energies, then the resulting $B$ mesons would be roughly stationary - making it hard to measure the decay time. Measuring the lifetime directly would be a difficult task. Instead, if a particle is boosted significantly with respect to the center of mass frame, a measurement of the distance a particle travels, 
and its velocity, allow one to calculate its lifetime $(\beta \gamma c \tau=d)$, where $d$ is the decay distance, $\tau$ is the lifetime, $\beta$ is the velocity/c and $\gamma$ is the relativistic boost. It was proposed by Pier Oddone of Lawrence Berkeley National Laboratory that if the electron and positron beams had different energies, then the resulting $B$ mesons would be moving as the center of mass after the collision would be moving/boosted [9], and hence easier to measure the small distances the $B$ mesons move. This is desirable, as then one only has to measure the position of production and decay of a $B$ meson to determine its lifetime. The PEP-II collider at SLAC stores an electron beam of energy $\sim 9.0 \mathrm{GeV}$ and a positron beam of $\sim 3.1 \mathrm{GeV}$. In this configuration, the $B$ mesons produced move at approximately half the speed of light and travel approximately 250 microns before decaying. This distance can be measured accurately but it requires a detector that is capable of measuring distances with an intrinsic resolution of $\sim 15 \mu \mathrm{m}$, in order to achieve a vertex resolution of approximately $80 \mu \mathrm{m}$.

\subsection{Summary}

After being first observed in the kaon system the origin of $C P$ violation is still a puzzle to physicists. It is hoped that the BABAR experiment will shed some light on this intriguing problem and determine whether its origins are indeed in the current Standard Model. 


\section{Chapter 3}

\section{The BaBar Detector}

The main goal of the BABAR experiment is to measure $C P$ asymmetries in the neutral $B$ meson system to determine if $C P$ violation originates in CKM mixing in the SM. To facilitate this, a new detector has been built at SLAC. The various collaboration members from each country were assigned specific parts of the overall detector. At this moment the completed sub-detectors are being fully assembled at SLAC. In the building/planning of such a detector, numerous criteria needed to be addressed:

- The need for an asymmetric detector. Since the two beams are of unequal energy the decay products are boosted forward in the laboratory frame.

- The fact that various machine elements have to come very close to the interaction region. Because of the high luminosities needed in the experiment unusual beam optics must be placed within close proximity of the interaction point where the two beams cross.

- A need for outstanding vertex resolution. The $B$ mesons travel roughly parallel to the z-axis so that the decay times are measured via the difference in their decay positions.

- To be able to perform tracking over a wide momentum range $\sim 60 \mathrm{MeV} / \mathrm{c}<$ $p_{t}<\sim 4 \mathrm{GeV} / \mathrm{c}$. 


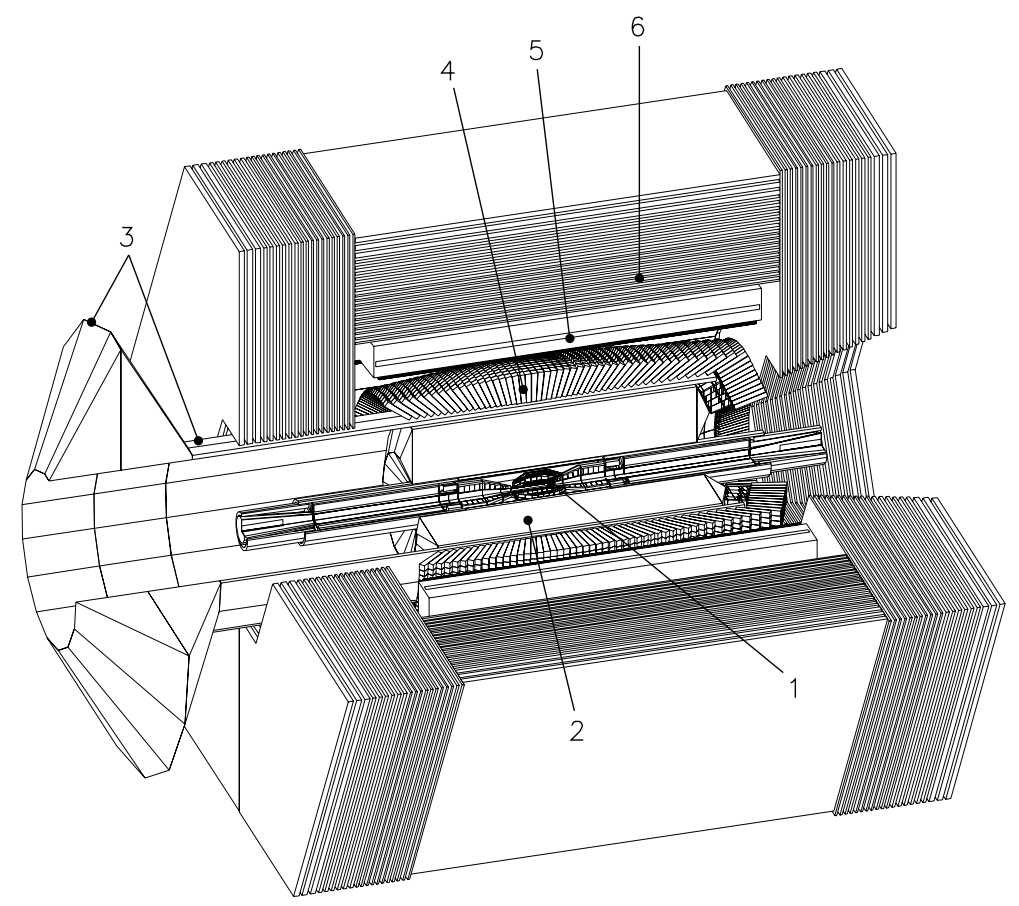

Figure 3.1: Layout of the BABAR detector. See text for key.

- To have the ability to discriminate between electrons, muons, pions, kaons and protons over a wide kinematic range. The process of tagging can only be done with a high efficiency if electrons, muons, and kaons can be identified. Also a $\pi-$ $K$ discrimination at high momentum $(2-4 \mathrm{GeV})$ is essential in order to distinguish the different decay channels $\left(B^{0} \rightarrow \pi^{+} \pi^{-}, B^{0} \rightarrow K^{ \pm} \pi^{\mp}, B^{0} \rightarrow \rho^{+} \pi^{-}, B^{0} \rightarrow K \rho\right.$, and $\left.B^{0} \rightarrow K \pi\right)$.

- To be able to detect photons and $\pi^{0}$ s over the wide energy range $\sim 20 \mathrm{MeV}<\mathrm{E}$ $<\sim 5 \mathrm{GeV}$.

- To have neutral hadron identification capability.

After all the above required criteria were assessed, a design was established and the detector constructed. A schematic of the detector is shown in Figure 3.1. The major 
subsystems of the detector are:

1. A Silicon Vertex Tracker (SVT). This provides precise position information on charged tracks and also is the sole tracking device for very low energy charged particles $\left(\operatorname{eg} p_{t}<100 \mathrm{MeV} / \mathrm{c}\right)$.

2. A Drift Chamber (DCH) filled with a helium-based gas, in order to minimize multiple scattering. This is responsible for the main momentum measurements for charged particles and helps in particle identification (PID) through energy loss measurements $(\mathrm{dE} / \mathrm{dx})$ and also contributes to the trigger.

3. A Detector of Internally Reflected Cherenkov light (DIRC). This is designed and optimized for charged hadron PID.

4. A Cesium Iodide Electromagnetic Calorimeter (EMC). It provides good identification and energy measurements for neutral particles as well as electrons down to an energy of about $0.5 \mathrm{GeV}$ as well as contributing to the trigger.

5. A superconducting coil, which provides a 1.5 T solenoidal magnetic field.

6. An Instrumented Flux Return (IFR) for muon identification down to about 0.6 $\mathrm{GeV}$ and neutral hadron PID.

The BABAR detector is extensively described in the Technical Design Report (TDR) [10]. This chapter serves only to highlight the main features of each sub-detector.

\subsection{The Silicon Vertex Tracker}

The main task of the vertex detector is to reconstruct the spatially separated decay vertices of the two primary $B$ mesons in order to determine the time between the two 


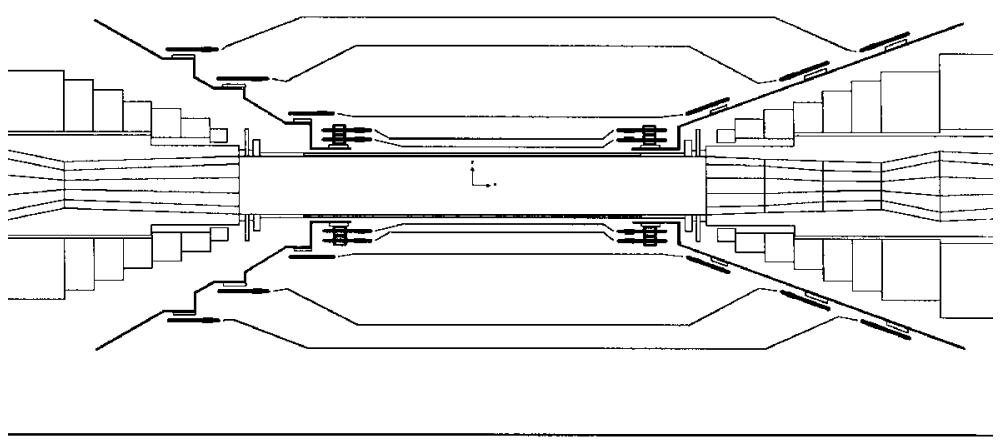

$10 \mathrm{~cm}$

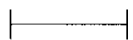

Figure 3.2: Detail of the inner part of the apparatus, showing the cross-sectional view of the silicon vertex tracker in a plane containing the beam axis.

decays. The SVT also provides excellent angular information as it is not so severely limited by the multiple scattering which plagues the other sub-detectors. For charged particles with transverse momenta less than $100 \mathrm{MeV} / \mathrm{c}$, the SVT supplies all the tracking information as such low momentum particles will not reach the drift chamber.

The basis on which BABAR hopes to observe $C P$ violation is to measure the separation between the two $B$ vertices with a precision better than one half the mean separation of $\sim 250 \mu m$ at PEP-II [11]. This works out to a precision of better than $80 \mu \mathrm{m}$ for a single vertex position measurement. Better precision would aid in pattern recognition, vertex reconstruction and background rejection, so the SVT is designed to have an intrinsic hit resolution of $10-15 \mu \mathrm{m}$ for the inner layers and $30-40 \mu \mathrm{m}$ for the outer ones.

Figures 3.2 and 3.3 show two different views of the Silicon Vertex Tracker. The SVT 


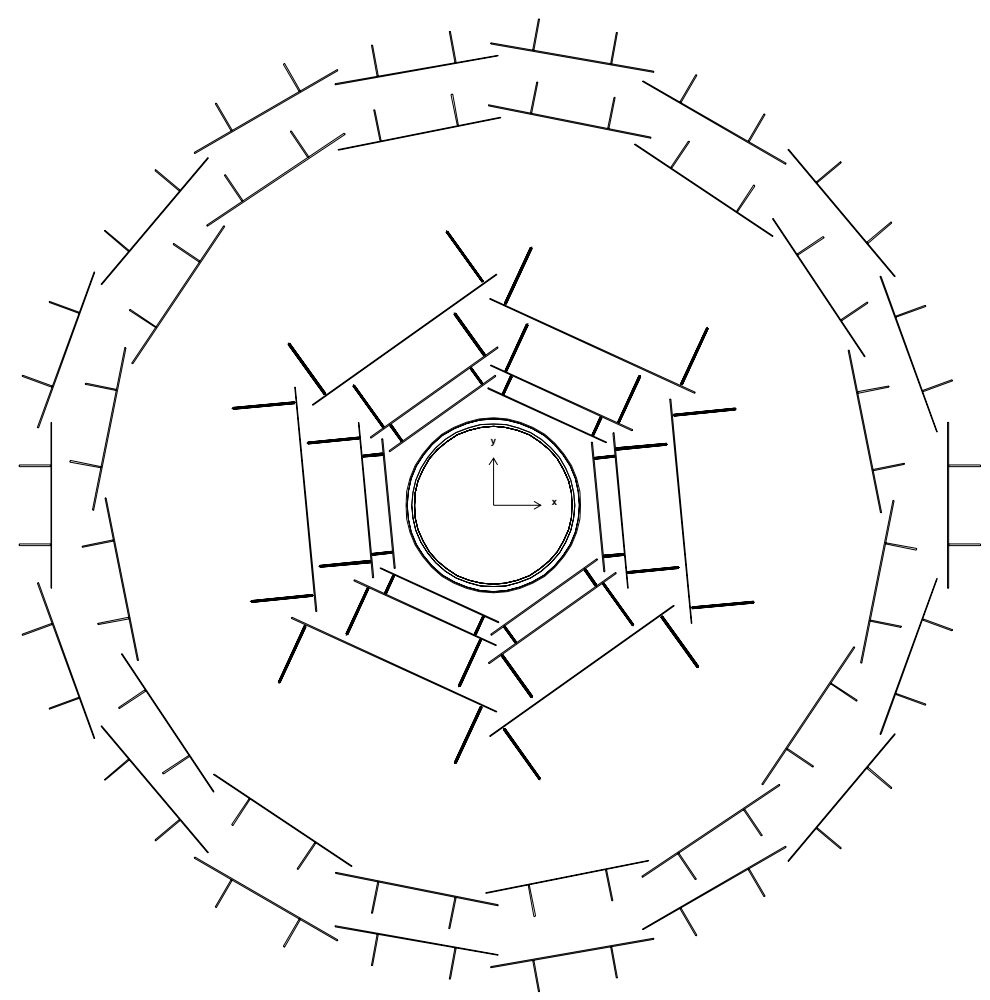

Figure 3.3: Layout of the BABAR silicon vertex tracker. Cross-sectional view in the plane orthogonal to the beam axis.

consists of five concentric cylindrical layers of double-sided silicon wafer detectors. Each layer is divided in azimuth into modules. The three inner layers have 6 detector modules. Layers 4 and 5 contain 16 and 18 detector modules respectively. The two outer layers are further divided into "a" and "b" modules, with "a"-type modules situated at a slightly smaller radius than the corresponding "b"-type ones (Fig 3.3), which allows the detectors to overlap. A pin-wheel type arrangement is employed in the inner modules to guarantee a similar overlap.

Each module is divided into two electrically separated forward and backward halfmodules. The inner sides of the detectors have strips oriented perpendicular to the 
beam direction to measure the $\mathrm{z}$ coordinate, while the outer sides have longitudinal strips, which allow for the $\phi$ coordinate measurement. The modules are supported on Kevlar ribs mounted to the end cones located in the forward and backward directions. A carbon-fiber space frame supports the assembly, which is mounted on the PEP-II final focusing magnets.

The BABAR SVT has 340 silicon detectors in total, covering an area of about $1 \mathrm{~m}^{2}$ with a total of $\sim 150,000$ readout channels.

\subsection{The Drift Chamber}

The drift chamber is the primary tracking device of the BABAR detector. It provides up to 40 measurements of space coordinates per track, which will ensure a high reconstruction efficiency for tracks with transverse momenta greater than $100 \mathrm{MeV} / \mathrm{c}$. The DCH performance goals are to provide spatial resolution better than $140 \mu m$, averaged over the cell in the $r-\phi$ plane, and to supply particle identification (PID) for low momentum tracks by $\mathrm{dE} / \mathrm{dx}^{1}$. For low momentum tracks, the momentum resolution is limited by the multiple coulomb scattering in the inner cylinder of the DCH as well as in the silicon vertex detector.

\subsubsection{The Drift Chamber Design}

A schematic view of the BABAR drift chamber is shown in figure 3.4. The DCH is a $280 \mathrm{~cm}$ long cylinder with inner radius of $23.6 \mathrm{~cm}$ and outer radius of $80.9 \mathrm{~cm}$. Since the collision products are boosted forward in the lab frame, the detector is designed to reduce the amount of material in the forward end. To accomplish this, the forward

\footnotetext{
${ }^{1} \mathrm{dE} / \mathrm{dx}$ is the energy loss per unit length for a particle traversing a particular medium.
} 


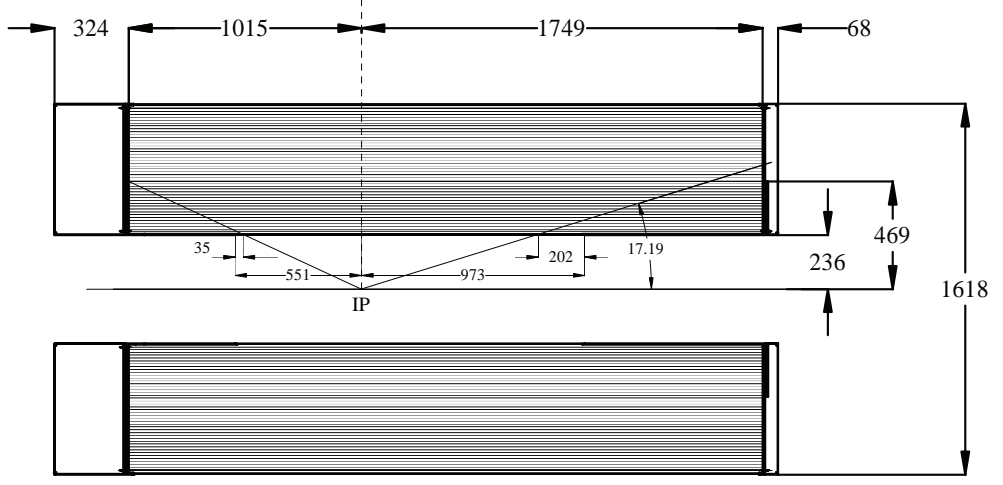

Figure 3.4: Side view of the BABAR drift chamber. The dimensions are in mm.

end plate is only $12 \mathrm{~mm}$ thick, compared to the rear end plate which has a thickness of $24 \mathrm{~mm}$. Also all the readout electronics and cooling apparatus are mounted on the rear end plate. The inner cylinder is made of $1 \mathrm{~mm}$ beryllium while the outer cylinder consists of 2 layers of carbon-fiber on a Nomex core.

The DCH cells are arranged in 10 superlayers of 4 layers each, giving a total of 40 layers. Axial (A) and stereo (U and V) superlayers alternate following the pattern AUVAUVAUVA as shown in figure 3.5. The stereo angle varies from a minimum of 40 mrad in the innermost stereo superlayer to a maximum of $70 \mathrm{mrad}$ in the outermost stereo superlayer. The 7104 cells are hexagonal in shape with typical dimension of $1.2 \times 1.8 \mathrm{~cm}^{2}$.

The sense wires are $20 \mu \mathrm{m}$ gold-plated tungsten-rhenium wiring while the field wires are $120 \mu \mathrm{m}$ and $80 \mu \mathrm{m}$ gold-plated aluminum. In order to keep the amount of material to a minimum, a gas mixture of Helium-isobutane (80\%:20\%) was chosen to fill the chamber. This mixture provides good spatial and $\mathrm{dE} / \mathrm{dx}$ resolutions and a reasonably 


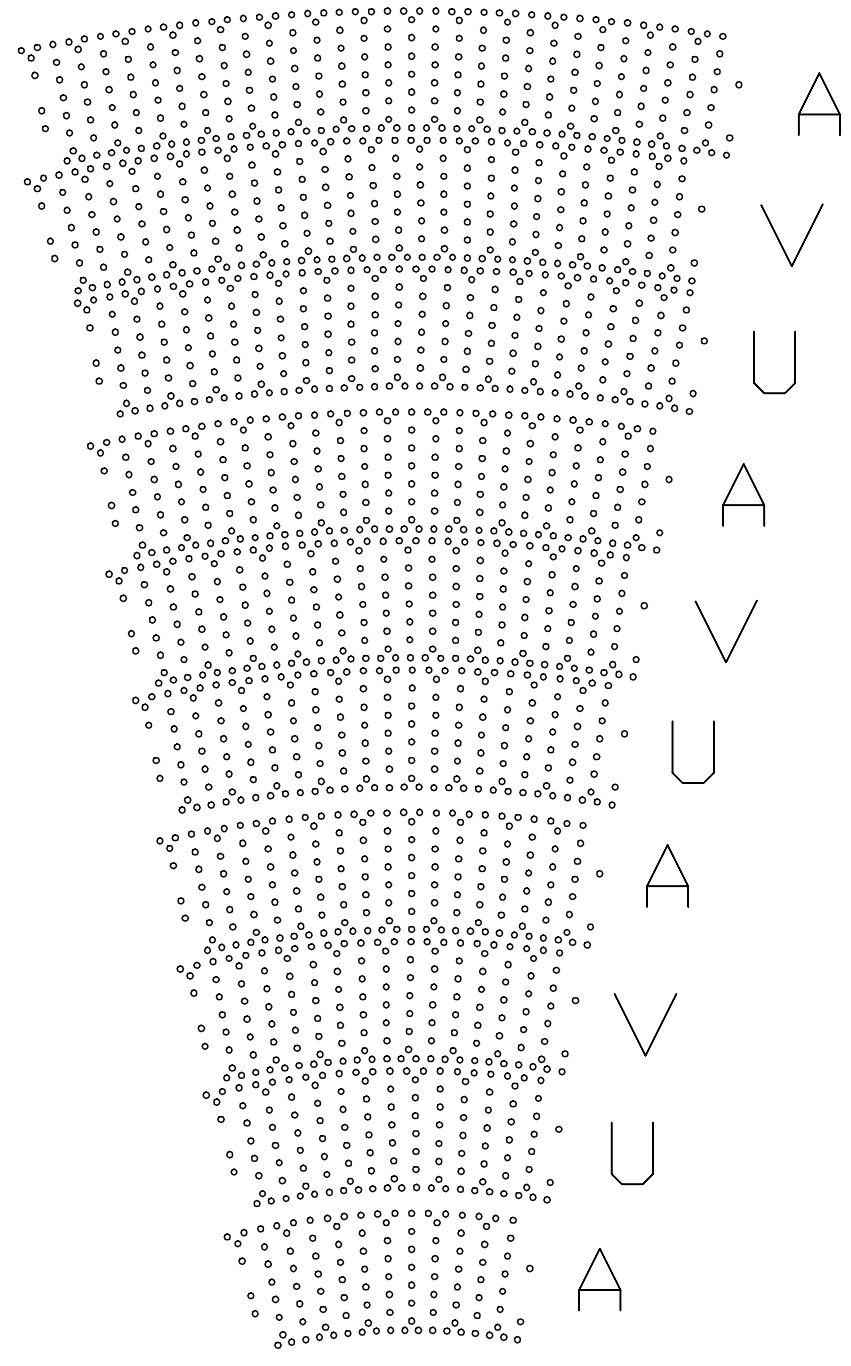

Figure 3.5: Cell layout in the BABAR drift chamber. 
short drift time. Nominal voltages of $1960 \mathrm{~V}$ for the sense wires, $340 \mathrm{~V}$ for the fieldshaping wires, and $900 \mathrm{~V}$ for the field wires at the boundaries of the superlayers are supplied by high voltage assemblies mounted on the rear end plate.

For drift-time measurements, the electronics detects the leading edge of the signal from the charge arriving at the sense wires and then digitizes the time with a $1 \mathrm{~ns}$ resolution. The $\mathrm{dE} / \mathrm{dx}$ measurements require integrating the total charge in the pulse.

\subsection{The DIRC}

The DIRC is a new type of Cherenkov-based detector devoted to PID. Specifically it is designed to provide excellent kaon identification for both tagging purposes and at high momenta for rare $B$ meson decays. To differentiate the two-body decay modes $B^{0} \rightarrow \pi^{+} \pi^{-}$and $B^{0} \rightarrow K^{+} \pi^{-}$, the DIRC must be able to separate pions from kaons up to about $4 \mathrm{GeV} / \mathrm{c}$, at large dip angles in the laboratory frame. The DIRC will also aid in muon identification in the low momentum range where the IFR is inefficient $(\lesssim 750 \mathrm{MeV} / \mathrm{c})$

The DIRC operates on the principle that charged particles traversing a DIRC quartz bar produce Cherenkov light. Through internal reflections the Cherenkov light is carried to the backward end of the bar (forward-going light is first reflected from a mirror located at the front end of the bar - see figure 3.6). The high optical quality of the quartz bar preserves the angle of the emitted Cherenkov light. The measurement of this angle, along with knowledge of track angle and momentum from the drift chamber, allows a determination of the particle velocity, from which its mass can be deduced. An advantage of the DIRC for an asymmetric collider is that the high momentum tracks are boosted forward, which causes a much higher light yield than for particles 


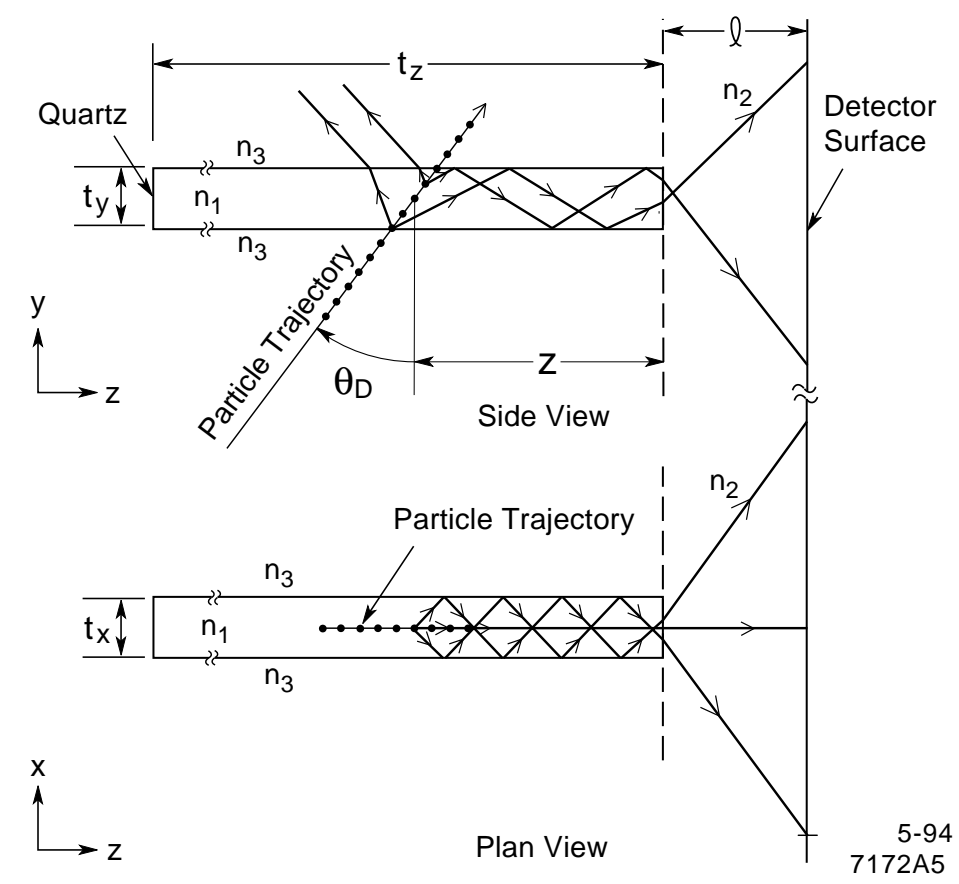

Figure 3.6: Schematic of a single radiator bar of a DIRC counter. The particle trajectory is shown as a connected line of dots; representative trajectories of Cherenkov photons are shown by lines with arrows.

at normal incidence. This is due to two effects: the longer path length in the quartz and a larger fraction of the produced light being internally reflected in the bar.

The DIRC is made up of 144 long straight polished bars of synthetic quartz with rectangular section, arranged in a 12-sided polygonal barrel. The DIRC radiator extends through the steel of the solenoid flux return in the backward direction to bring the Cherenkov light outside the tracking and magnetic volumes.

At the instrumented end of the quartz bars, the Cherenkov image is permitted to expand and be projected into a tank of purified water, called the Standoff Box. Water was chosen because its refractive index matches pretty closely to that of the quartz 


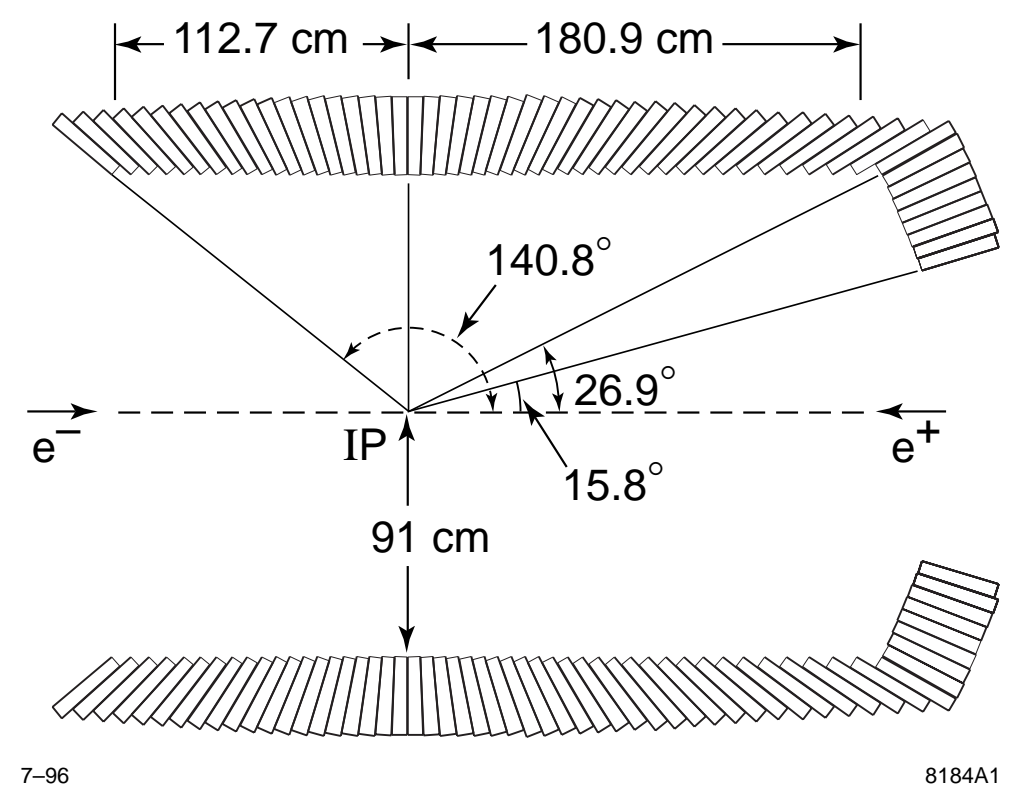

Figure 3.7: The EMC layout: Side view showing dimensions (in mm) of the calorimeter barrel and forward endcap.

bars, thus minimizing the total internal reflection at the quartz-water interface. The Standoff Box contains approximately 11,000 photo multiplier tubes (PMTs) to cover the detector area. The PMTs are located about $1.2 \mathrm{~m}$ from the end of the quartz bars. The phototubes are located in a gas-tight volume as protection against helium leaks from the drift chamber. The DIRC occupies only $8 \mathrm{~cm}$ in radial direction of the detector, which allows for a relatively large radius for the drift chamber while at the same time keeping the volume of the CsI Calorimeter reasonably low.

\subsection{The Electromagnetic Calorimeter}

The primary physics goals of the BABAR detector, which require the tagging and reconstruction of $B$ events containing $C P$ eigenstate decays, are ideally suited to Cesium 
Iodide (CsI) calorimetry. The need to effectively and cleanly reconstruct $C P$ eigenstates containing one or more $\pi^{0}$ decays makes excellent electromagnetic calorimetry essential. High efficiency for low energy photons is also required to offset the small branching fractions typical of most $C P$ eigenstates and to make it possible to reconstruct final states containing several $\pi^{0} \mathrm{~s}$. By facilitating lepton identification $(e / \pi$ and $e / \mu$ separation) the calorimeter also provides one of the tags needed for every $C P$ analysis.

The calorimeter consists of a cylindrical barrel section and a forward conic endcap (Figure 3.7). Radially, the barrel is located outside the DIRC system and within the magnet cryostat. The barrel has an inner radius of $91 \mathrm{~cm}$ and an outer radius of 136 $\mathrm{cm}$. It is located asymmetrically about the interaction point. The barrel consists of $5760 \mathrm{CsI}(\mathrm{Tl})$ crystals, arranged in 48 polar-angle $(\theta)$ rows of distinct crystal sizes, each having 120 identical crystals in azimuthal angle $(\phi)$.

The forward endcap is a conic section with front and back surfaces tilted $22.7^{\circ}$ with respect to the vertical. It contains $820 \mathrm{CsI}(\mathrm{Tl})$ crystals, arranged in $8 \phi$ rings, starting at an inner radius of $55.3 \mathrm{~cm}$ from the beam line. In order to reduce the amount of inactive material in front of the detector all the cooling, cables, and services are located at the back.

\subsection{The Instrumented Flux Return}

Muon identification and neutral hadron detection are provided by the Instrumented Flux Return, which makes use of the large iron structure needed as the magnet return yoke. It is segmented and instrumented with Resistive Plate Counters (RPCs) and consists of a central part (Barrel) and two plugs (End Caps) (figure 3.8). 


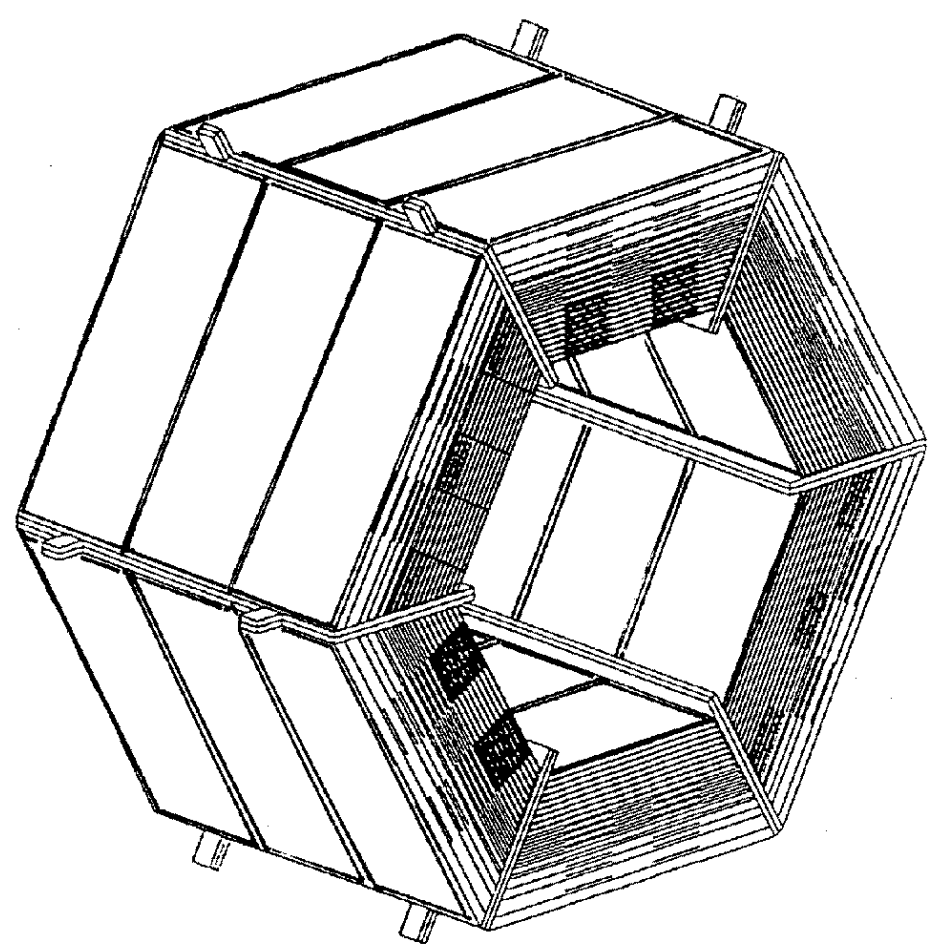

Figure 3.8: The IFR Barrel: layout of the RPC Modules.

An interesting feature of the BABAR experiment is the graded segmentation of the iron, which varies from $2-10 \mathrm{~cm}$, increasing with the radial distance from the interaction region. This segmentation is the result of detailed Monte Carlo studies which have shown that muon identification at low momentum and $K_{L}^{0}$ detection improve, for a given amount of absorber, as the thickness of the iron plates decreases. This effect is most important in the first absorption length, so that grading the segmentation improves the performance without increasing the number of layers significantly. The iron is segmented into 18 plates, giving a total thickness of $65 \mathrm{~cm}$ in the Barrel and $60 \mathrm{~cm}$ in the End Caps. Each End Cap door has a hexagonal shape and is divided vertically into 2 halves to allow access to the inner detectors. 
The main goal of the IFR detector is to achieve the highest practical muon tagging efficiency. About $18 \%$ of all $B$ decays contain at least one muon in the region covered by the BABAR detector. Charged tracks found in the DCH will be matched with segments in the IFR and be identified using a detailed analysis of the hit patterns in the active detectors. Neutral hadron ( $K_{L}^{0}$ and neutrons) identification will result from a detailed analysis of the hit patterns in the active detectors with no track matches from the tracking chambers.

\subsection{Summary}

The BABAR detector has taken years to plan and build. This effort will be justified by the observation (though not guaranteed, a non-observation of $C P$ asymmetry would also have strong implications on the $\mathrm{SM}$ ) and understanding of $C P$ violating processes in the neutral $B$ meson system. But the physical detector is only the first step in the journey of observing, cataloging, and fully understanding the origin of $C P$ violation. The output from the various sub-detector components are used as input in the reconstruction software. This software is responsible for taking all the electronic information and making an accurate representation of what actually happened in the detector. It is only after this is done can one see if $C P$ violation has occurred. 


\section{Chapter 4}

\section{The Drift Chamber}

Drift chambers are used to detect and measure ionizing radiation. This radiation is the result of charged particles passing through the chamber and interacting with the gas contained inside. The three original gas type detectors were the ionization chamber, the proportional counter and the Geiger-Müller counter. Not until the late 1960's with the invention of the multi-wire proportional chamber did these gas ionization instruments become a integral part of high energy physics experiments. From this renewed interest came the development of the drift chamber as well as the time projection chamber. Although employing the same basic principles as the proportional counter, these two new detectors bore little resemblance to their simple predecessor.

\subsection{Ionization Detectors}

All ionization detectors work in essentially the same way. The detectors are composed of cells. A wire running through the center of the cell, called the anode or sense wire, is maintained at a positive voltage relative to the wires that surround it. These surrounding wires are called cathode or field wires and together with the anode create an electric field directed from the anode to the cathode.

When a high energy particle traverses the chamber it has the potential to create 


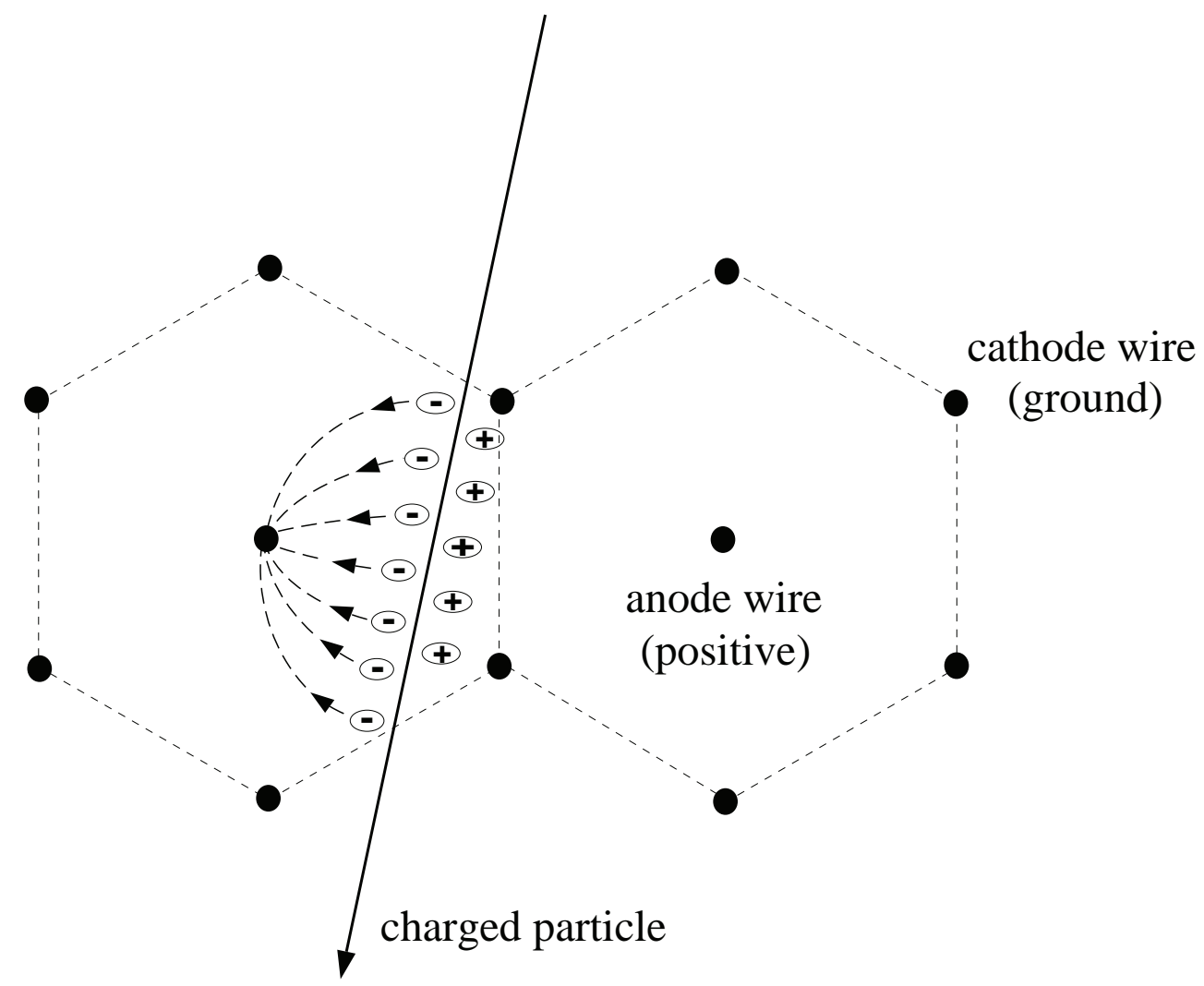

Figure 4.1: Side view of the BABAR drift chamber. The dimensions are in $\mathrm{mm}$.

electron-ion pairs (Figure 4.1) . This process is referred to as ionization and is a result of the particle colliding with one of the atoms in the gas, releasing an electron. In an electric field, the negatively charged electrons will then drift toward the nearest anode wire because of its positive potential. The remaining ions will move toward the cathode wires which are kept at a negative potential relative to the anode ones.

The anode wires in a drift chamber have a high voltage applied to them. The reasoning for this is that it causes the newly freed electrons to accelerate toward the anode wire. Due to this high acceleration, each of the original or primary electrons is then able to gain sufficient energy to further ionize the gas molecules that lie in its path. These 
secondary electrons can then repeat the process, increasing the number of electrons that are moving toward the anode wire. This procedure escalates and is referred to as an ionization avalanche. If there are enough electrons in the avalanche that are collected at the anode wire then a signal may be detected on the wire. The number of electrons that are collected on the sense wire is directly proportional to the energy of the charged particle that produced the primary electron. The gas gain is defined to be the ratio of the number of electrons in the avalanche that arrive at the sense wire to the number of initial primary ions, and is typically on the order of $10^{6}[12]$.

The time between the first ionization at time $t_{0}$, and the arrival of the signal at the wire at time, $t$, can be measured using an external trigger $\left(t_{0}\right.$ is also obtained from an iteration fit of all the tracks in an event). Using this information the drift time can be determined. If the electrons drift toward the sense wire with a known velocity, $v_{d}$, called the drift velocity, then the drift distance of the particle to the wire when it passed through the cell can also be calculated:

$$
x=\int_{t_{0}}^{t} v_{d} d t .
$$

How well the position of the particle is known and the detection efficiency is primarily governed by the choice of gas and the cell size and geometry used in the chamber.

\subsection{Gas Properties}

Numerous gas properties dictate whether a certain gas is appropriate to fill a drift chamber. A few of these characteristics include the type of gas, drift velocities and how the quality for various gases reflect on chamber stability. 


\subsection{Gas Type Choice}

The specific requirements for the BABAR drift chamber were outlined in Chapter 3. These included a gas that minimized multiple Coulomb scattering and provided good spatial and $\mathrm{dE} / \mathrm{dx}$ resolutions with a reasonably short drift time. Although important for this particular experiment, there are a number of more generic issues one faces when deciding what gas to use to fill a drift chamber.

A drift chamber operating at room temperature can only be filled with elements that are in a gaseous state. These include oxygen $\left(\mathrm{O}_{2}\right)$, nitrogen $\left(\mathrm{N}_{2}\right)$, fluorine $\left(\mathrm{F}_{2}\right)$, and the noble gases, helium (He), neon (Ne), argon (Ar), krypton (Kr), and xenon (Xe). In the above list only the noble gases are monatomic while the others (oxygen, nitrogen and fluorine) are diatomic. Diatomic molecules are more complex than monatomic ones and if molecular size is an issue, then this needs to be addressed.

A main function of the gas in a drift chamber is to aid in the production of an ionization avalanche. Depending on whether the gas is monatomic or diatomic, this avalanche can occur at different operating anode voltages. This avalanche arises in noble gases at a much lower anode voltage than with gas mixtures comprised of complex diatomic molecules. Due to the numerous non-ionizing energy dissipative modes present in the diatomic molecules, they are able disperse energy through collisions or disassocations into smaller molecules. These modes are not accessible in the noble gas molecules which can only return to their ground states by the release of a photon. This photon is then able to continue the process of ionization avalanche and hence a lower anode voltage is needed to obtain a sufficiently large pulse arriving at the wire.

At high voltages there is a problem with having only noble gases present in the chamber. 
The photon released during a noble gases de-excitation is ultraviolet. As a consequence, it possesses an energy (11.6 eV for argon, $19.8 \mathrm{eV}$ for helium)[13] that is greater than the work function ${ }^{1}$ of any metal cathode $(4.82 \mathrm{eV}$ for a gold plated cathode)[14]. These newly freed electrons then repeat the process of ionization on their journey toward the anode. The solution to this dilemma is to add what is called a quencher gas. This quencher gas is a complex molecule, such as carbon dioxide $\left(\mathrm{CO}_{2}\right)$ or iso-butane $(i-$ $\mathrm{C}_{4} \mathrm{H}_{10}$ ), that retards the ejection of these unwanted electrons from the cathode wires. This is accomplished because these complex molecules have a large number of nonradiative excited states - both rotational and vibrational. Utilizing these states, it is possible for a complex molecule to absorb and then dissipate a wide range of photon energies, thereby eliminating the ultraviolet radiation before it reaches the cathode. Since these modes do not result in a production of an electron, the extra ionization process is stopped from occurring.

Although the quencher gas solves one problem it creates another, the process of wire aging. This occurs when liquid or solid polymer deposits begin to form on the chamber wires, seriously affecting the operation of the drift chamber [15].

\subsubsection{Electron Diffusion}

The limit on the spatial resolution of a drift chamber is governed predominantly by the diffusion of electrons in the chamber medium as they move toward the anode wire [13]. In the absence of an electric field, the electron-ion pair formed by the particle diffuses uniformly outward, from regions of high concentration to regions of low concentration from their point of creation. The concentration of electrons at a

\footnotetext{
${ }^{1}$ The work function of a metal is the energy that a photon needs in order to just free one of the outermost electrons.
} 
later position $x$ after diffusing at time $t$ is approximately Gaussian, and is given by the following expression [13]:

$$
\frac{d N}{d x}=\frac{N_{0}}{\sqrt{4 \pi D t}} \exp \left(-\frac{x^{2}}{4 D t}\right)
$$

where $x$ is the distance from the point of ionization, $N_{0}$ is the initial number of electrons at $x=0$ and $t=0$, and $D$ is the diffusion coefficient which, from kinetic theory, is given by:

$$
D=\left(\frac{u^{2}}{3 \nu}\right)_{\text {average }}=\frac{1}{3} l \bar{u}
$$

where $\bar{u}$ is the mean thermal velocity of the electron discharged by the incoming ionizing particle, $\nu$ is the frequency of electron collisions with the chamber gas and $l$ is the mean distance that the electron travels before colliding with an atom or molecule in the chamber medium (called the mean free path).

Comparing Equation 4.2 to the form of a Gaussian distribution, distributed about $x=0$ :

$$
y=\frac{1}{\sigma \sqrt{2 \pi}} \exp \left(-\frac{x^{2}}{2 \sigma^{2}}\right)
$$

the standard deviation of the electron distribution, or the spatial spread of electrons at $x$ is identified as:

$$
\sigma(x)=\sqrt{2 D t}=\sqrt{2 D \cdot \frac{l}{v_{d}(x)}}=\sqrt{\frac{m_{e} \bar{u}^{2}}{e E(x)}},
$$

where $E(x)$ is the electric field, $e$ is the charge of an electron and $m_{e}$ is the electron mass. The dependence of spatial resolution on the size of the cell is now explicitly clear. The diffusion distribution varies as the square root of the drift length, which means that the longer the path the electron travels, the more diffuse the distribution will be due to an increase in the number of collisions. Ultimately, a high diffusion will adversely affect the spatial resolution of a drift chamber since the timing for a 
dispersed group of electrons becomes difficult to determine accurately. Therefore, a small cell size should give improved spatial resolution as well as avoid the use of very high voltages. With drift path lengths on the order of $1 \mathrm{~cm}$, spatial resolution values of less than $100 \mu \mathrm{m}$ are expected [17].

\subsubsection{Drift Velocity}

The drift velocity, $v_{d}$, of an electron superimposed on random thermal motion in the presence of an electric field, is given by the expression [16]:

$$
v_{d}(x)=\frac{2}{3} \frac{e l}{m_{e} \bar{u}} E(x)
$$

Low drift velocities lead to large drift times and therefore better position resolution because of the improved timing resolution. In experiments such as BABAR where there is expected to be a high count rate, a high drift velocity is required as one has to worry about deadtime ${ }^{2}$ in the drift chamber.

\subsection{A Drift Chamber in a Magnetic Field}

Another method of gaining more information from the drift chamber is by placing it in a magnetic field. The Lorentz force, F, is defined as,

$$
\mathbf{F}=q \mathbf{v} \times \mathbf{B}
$$

where $\mathbf{B}$ is a uniform magnetic field. The magnetic field deflects charged particles that have a component of velocity perpendicular to $\mathbf{B}$ causing them to trace out a helix as they propagate along the z-axis. The radius of curvature, $r$, for this helix is given by,

$$
r=\frac{m v \sin \theta}{q B}=\frac{p \sin \theta}{q B}
$$

\footnotetext{
${ }^{2}$ This is the finite time required by the detector to process an event during which time it is unable to react to any other signals.
} 


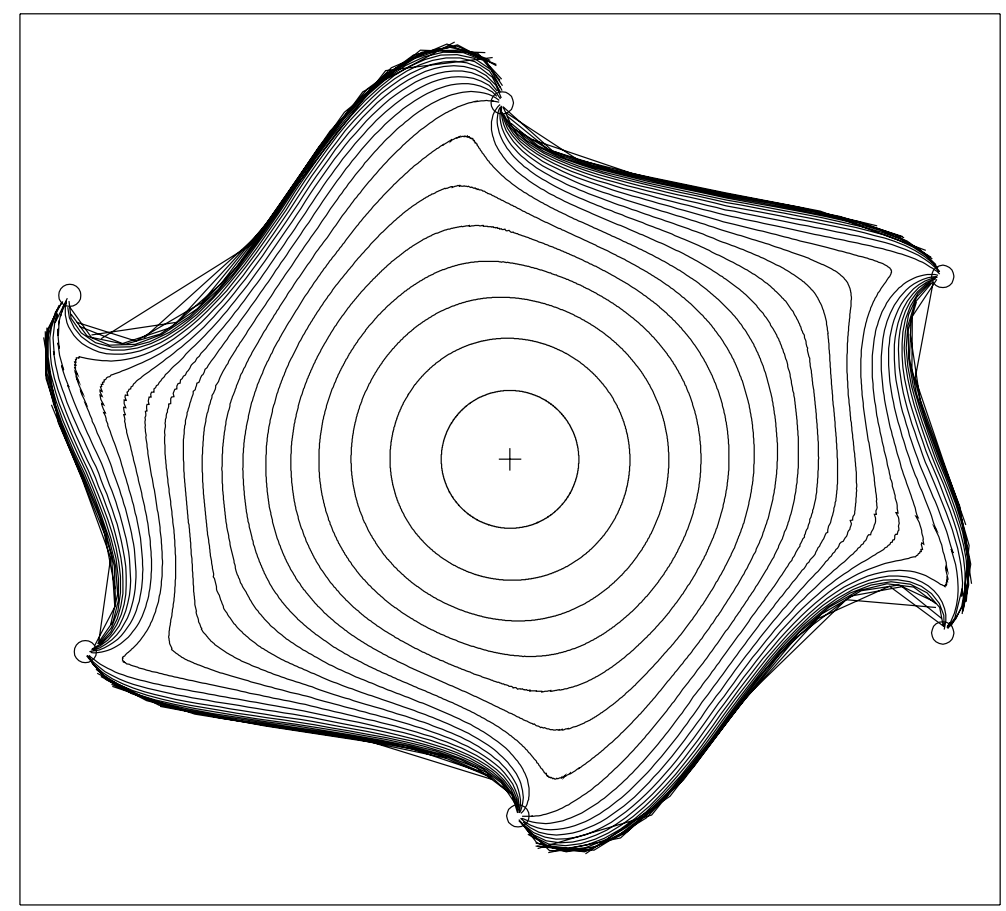

Figure 4.2: 50 ns isochrones in a typical BABAR drift chamber cell.

where $q$ is the charge of the particle. Taking the expression for the transverse momentum, $p_{t} \equiv p \sin \theta$, together with Equation 4.8 gives,

$$
p_{t}=r q B
$$

So if one knows the magnetic field, then one can determine the radius of curvature and hence the momentum of a charged particle in the drift chamber. The BABAR experiment uses a $1.5 \mathrm{~T}$ magnetic field which has been extensively studied. Figure 4.2 shows the $50 \mathrm{~ns}$ isochrones in a typical cell of the drift chamber. 


\subsection{Summary}

The advent of the drift chamber has meant that it is now possible to measure the trajectories of particles in high energy physics experiments with great precision. By careful consideration on the choice of gas type, cell size and drift velocity, one can localize particles to distances of $\mathcal{O}\left(10^{-4} \mathrm{~m}\right)$. In the BABAR experiment this increased accuracy will aid in pattern recognition, and will be used in $\mathrm{dE} / \mathrm{dx}$ calculations. 


\section{Chapter 5}

\section{The Silicon Vertex Tracker}

The silicon vertex tracker is a type of detector based on the crystalline semiconductor silicon. Work on crystal detectors was performed back in the early 1930's [18] but it was not until the late 1950's that real development on these instruments began. The basic operating principle of a semiconductor detector is analogous to gas ionization

detectors. Instead of a gas, the medium is now a solid semiconductor material. Passing ionizing radiation creates electron-hole pairs (instead of electron-ion pairs) which are then collected by an electric field. The average energy required to create an electronhole pair is about 10 times smaller than the energy needed for gas ionization. This advantage leads to a better energy resolution. Because they are crystalline materials, semiconductor detectors have a greater sensitivity to radiation, which limits their long term use. To fully understand the operation of the SVT, one first needs a brief overview of semiconductor properties along with a general outline of semiconductor detectors.

\subsection{Basic Semiconductor Properties}

A semiconductor is a crystalline material whose outer shell atomic levels display an energy band structure [13]. This structure consists of a valence band, a "forbidden" energy gap and a conduction band (Figure 5.1). The energy bands are actually regions 


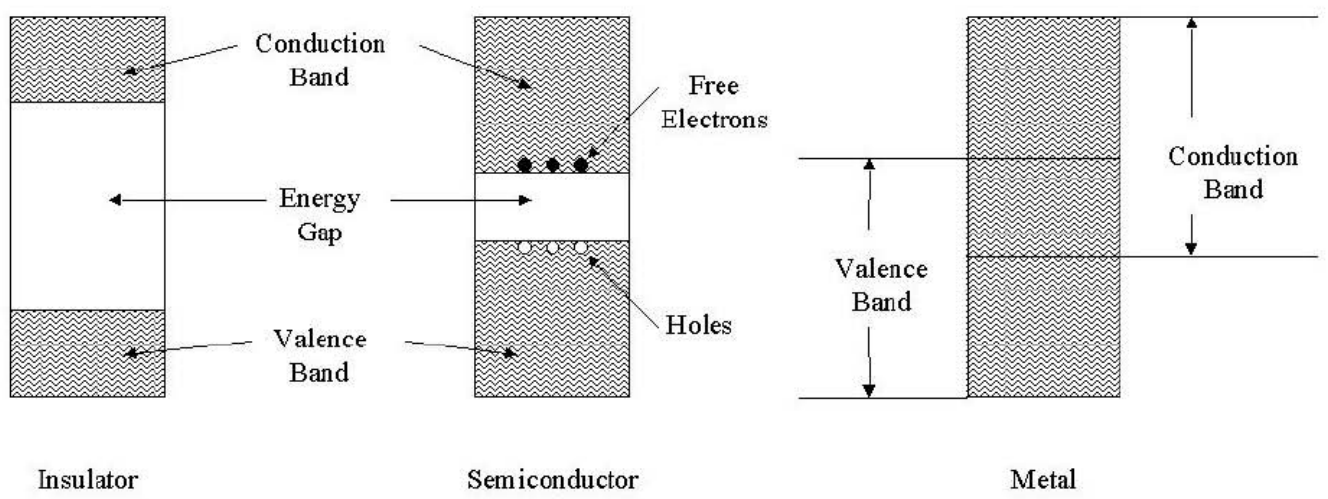

Figure 5.1: Energy band structure of insulators, semiconductors, and metals.

of closely spaced energy levels which are close enough to be regarded as a continuum. The "forbidden" energy gap is a region devoid of energy levels. The conduction band is the highest energy band. In this region, electrons are detached from their parent atoms and are free to roam the entire crystal. In contrast, the valence band electrons are more tightly bound and remain associated with their respective atoms.

The width of the gap and bands is determined by the lattice spacing between the atoms. In a conductor the energy gap is nonexistent, while for insulators the gap is large. In a semiconductor, the energy gap is intermediate in size so that only a few electrons are excited into the conduction band by thermal energy. Therefore, when an electric field is applied, a small current is produced.

\subsubsection{Charge Carriers}

Thermal energy can excite a valence electron into the conduction band, thereby leaving a hole in its original position. In such a state the neighbouring valence electron will 
jump from its bond to fill this hole. This leaves a hole in the now neighbouring position. If the next neighbouring electron repeats the process and so on, then the hole appears to move through the crystal. The hole is positive relative to the sea of negative electrons in the valence band and its movement through the crystal creates an electric current. In a semiconductor, the electric current arises from two sources: the movement of free electrons in the conduction band and the movement of holes in the valence band.

If the major charge carriers are the electrons then the semiconductor is referred to as a n-type semiconductor. If on the other hand the holes are the ones carrying most of the charge then the semiconductor is called a $p$-type semiconductor.

\subsubsection{Intrinsic Charge Carrier Concentration}

As stated above, electron-hole pairs are constantly being generated by thermal energy. Simultaneously, there are a certain number of electrons and holes which recombine. Under stable conditions, an equilibrium concentration of electron-hole pairs is established. If $n_{i}$ is the concentration of electrons (or holes) and $T$ is the temperature then,

$$
n_{i}=\sqrt{N_{c} N_{v}} \exp \left(\frac{-E_{g}}{2 k T}\right)=A T^{3 / 2} \exp \left(\frac{-E_{g}}{2 k T}\right)
$$

where $N_{c}$ is the number of states in the conduction band, $N_{v}$ is the number of states in the valence band, $E_{g}$ is the energy gap at $0 \mathrm{~K}, k$ is the Boltzmann constant and $A$ is a constant independent of $T$. Typical values of $n_{i}$ are on the order of $1.5 \times 10^{10} \mathrm{~cm}^{-3}$ for silicon at $T=300 \mathrm{~K}$. 


\subsubsection{Mobility}

The drift velocity of the electrons and holes through a semiconductor under the action of an externally applied electric field are

$$
\begin{aligned}
& v_{e}=\mu_{e} E \\
& v_{h}=\mu_{h} E
\end{aligned}
$$

where $E$ is the magnitude of the electric field and $\mu_{e}$ and $\mu_{h}$ are the mobilities of the electrons and holes respectively. For a given material, the mobilities are functions of $E$ and the temperature $T$. For silicon at normal temperatures [19], $\mu_{e}$ and $\mu_{h}$ are constant for $E<10^{3} \mathrm{~V} / \mathrm{cm}$, thereby making the relationship between velocity and $E$ linear. For $E$ between $10^{3}-10^{4} \mathrm{~V} / \mathrm{cm}, \mu$ varies approximately as $E^{-1 / 2}$, while for $E$ $>10^{4} \mathrm{~V} / \mathrm{cm}, \mu$ varies as $E^{-1}$. At these high $E$ values the velocity saturates, due in part to a proportional fraction of the kinetic energy acquired by the electrons and holes being drained by collisions with the lattice atoms.

For temperatures between $100 \mathrm{~K}$ and $400 \mathrm{~K}, \mu$ also varies approximately as $T^{-m}$. In silicon $m=2.5$ for electrons and $m=2.7$ for holes.

The current in a semiconductor is determined by the mobilities. The current density $J=\rho v$, where $\rho$ is the charge density and $v$ is the velocity in a semiconductor is given by

$$
J=e n_{i}\left(\mu_{e}+\mu_{h}\right) E
$$

where the fact that current is carried by both electrons and holes has been used. 


\subsection{The np Semiconductor Junction}

All present day semiconductor detectors depend on the formation of a semiconductor junction to function. A simple junction is the np junction which is formed by the juxtaposition of a n-type material and a p-type semiconductor. One method of achieving this is to diffuse sufficient p-type impurities into one end of a homogeneous bar of n-type material so as to change that end into a p-type semiconductor [13].

The np junction creates a special zone around the interface between the two materials. Due to the difference in the concentration of electrons and holes between the two materials, there is an initial diffusion of holes toward the n-region and a corresponding diffusion of electrons toward the p-region. Consequently, the diffusing electrons fill up holes in the p-region while the holes capture electrons in the n-region (figure 5.2). Although initially neutral, the $\mathrm{n}$ and $\mathrm{p}$ structures begin to develop a definite charge. Being injected with extra electrons, the p-region becomes negative while the n-region becomes positive. This creates an electric field across the junction that eventually stops the diffusion process. As a result a region of immobile charge is created referred to as the depletion zone. The electric field gives rise to a potential difference across the junction, called the contact potential. Any electron or hole created or entering the depletion zone is swept out by the electric field. If ionizing radiation enters this zone and produces an electron-hole pair then a current signal proportional to the ionization will be detected by placing contacts on either end of the junction. 


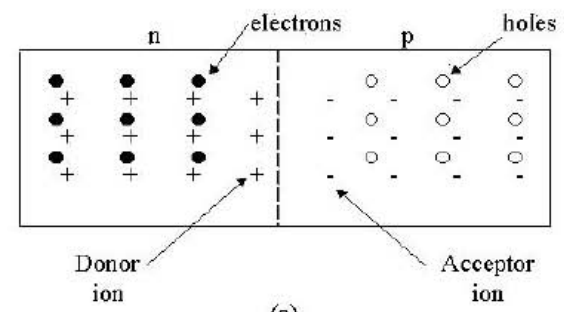

(a)

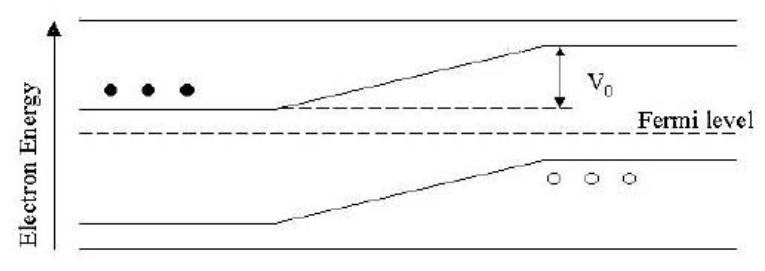

(b)

Figure 5.2: (a) Schematic diagram of an np junction, (b) Diagram of electron energy levels showing creation of a contact potential $V_{0}$.

\subsection{Micro-Strip Detectors}

In the 1980's, there was a renewed interest in using semiconductor detectors in high energy physics. This was due in part to a demonstration that showed a $5 \mu \mathrm{m}$ spatial resolution using a silicon micro-strip detector [20]. Such a device is composed of separate readout strips arranged at very small intervals, $\mathcal{O}(10 \mu \mathrm{m})$. The micro-strip detector that BABAR is using use double-sided silicon strips, AC coupled with polysilicon bias resistors [21]. Six different types of detectors are needed (I to VI) in BABAR, each having a different physical dimension, number of strips and readout pitches. The "barrel" portion of the detector (I to V) have orthogonal strips on two sides. The strips responsible for reading out the $z$ coordinate are on the junction side for the model I, II, and III detectors, and on the ohmic side for models IV and V. The strip on the "wedge" detectors are not orthogonal on the two sides. The ohmic side, responsible for measuring the $z$ coordinate, has strips that with a constant width and pitch. The junction side has strips having different starting and ending pitches and is responsible for reading out the $\phi$ coordinate. 


\subsection{Summary}

Semiconductor detectors have advanced along way since they were used primarily for energy measurements. BABAR is employing a silicon detector with an intrinsic resolution of $\sim 10 \mu \mathrm{m}$. This great precision will aid the in the position measurements of the decaying particles in the BABAR detector and will be a valuable asset in the search for $C P$ asymmetry. 


\section{Chapter 6}

\section{Tracking Reconstruction}

The most important role of the tracking reconstruction is finding charged tracks and providing the most accurate possible estimate of the physics parameters of the underlying charged particle. Efficiency of close to $100 \%$ in $C P$ physics events that have a transverse momenta, $p_{t}$, greater than $50 \mathrm{MeV} / \mathrm{c}$ is required within the acceptance of the Silicon Vertex Tracker and the Drift Chamber. Along with the $C P$ physics requirements are:

1. Documentation of the efficiency of tracking. This includes information on rates of lost and unphysical tracks, two track resolutions, etc.

2. Provide precise position and angular information to the DIRC, EMC, and IFR, with accuracy consistent with their resolution.

3. Provide an estimate of the position of the beam spot, and do production primary vertex finding.

In addition, many detector sub-systems require an accurate estimate of in which bunch a given event originated. Although this will involve the use of detailed information from other systems, responsibility for this is placed with the DCH and tracking software because of need and opportunity. 


\subsection{Reconstruction}

The BABAR reconstruction software [22] is organized as a set of Modules, which process sequentially the data in an Event. The execution of these modules is controlled by a Framework, which provides a command line facility for controlling execution and access to the Tcl/Tk scripting language. A typical reconstruction package usually consists of approximately 60 modules, arranged in Sequences to do subdetector-specific processing (hit finding, etc.), followed by pattern recognition, fitting, and subdetectorspecific particle identification.

Eventually the results of these computations will be saved in an event store that is currently being constructed. During this construction phase another means of data storage needed to be developed. The BABAR Event Analysis Summary Tapes (Beast tapes) were the solution that was used. Utilizing these tapes meant that certain of the high-level reconstruction results could be stored after computation and read back into simple analysis software.

The output of reconstruction is accessed through an analysis toolkit called Beta [23]. Beta provides code enabling the analyst to treat charged tracks and clusters as fourvectors. It also provides access to vertexing, particle identification, Monte-Carlo simulation truth information, and reconstruction of sequential decay chains. Beta can be used with either the output of the fast simulation, Aslund, the summary tapes (Beast), or the full reconstruction. In this way, the same analysis program can generally be used for each kind of input. 


\subsubsection{Tracking}

Before a charged particle trajectory can be reconstructed it must first be tracked. What this entails can be broken down into two stages, pattern recognition and fitting.

\section{Pattern Recognition}

The two tracking devices, the silicon vertex detector and the drift chamber, each use their own specific track finding algorithms to locate tracks. The silicon vertex detector algorithm first combines $r-\phi$ and $z$ hits in the same silicon wafer to form space points. It then does an exhaustive search for good helical tracks, requiring hits in at least four out of the five layers of silicon.

Two drift chamber algorithms are run in sequence. The first drift chamber algorithm

finds straight-line track segments in all ten superlayers. It then combines segments to form first a circular track using $\mathrm{r}$ and $\phi$ information (using axial segments only, and with a strong bias towards tracks coming from the interaction point), and then a helical track (by adding stereo segments to the axial track).

The second drift chamber tracking-finding algorithm uses 2D circular superlayer segments in three adjacent superlayers (all eight possibilities are tried) to form a trial helix. If a helix of sufficient quality is found, it is projected forward and backward, and other segments are added. This algorithm is designed to find tracks not coming from the primary vertex (such as $K_{S}^{0}$ and other decays), tracks which only pass through a small number of superlayers (large dip angle tracks), and low $p_{t}$ tracks (loopers).

Another piece of code merges the separately-found drift chamber and silicon vertex detector tracks. It projects each silicon vertex detector and drift chamber track to the 
support tube and looks for good matches. Those that match are combined into a single track, which is placed in the output list of good tracks.

\section{Fitting}

After pattern recognition, all tracks are initially fitted with a simple helix fitter, which ignores interactions with materials. After merging of drift chamber and silicon vertex detector tracks, the merged tracks are assigned track parameters based on a weighted average of the two input tracks. All tracks in the output list (merged or not) are then refitted with a Kalman filter fitter, using as a mass hypothesis the mass of the pion. The effects of multiple scattering and energy loss in the detector are included in the fit. The detector description used in the calculation of material effects includes a fairly detailed model of the vertex detector, including support structure, models of the beampipe and support tube, and a simple model of the drift chamber (treating the gas as a set of concentric cylinders of homogeneous wire/gas mixture).

\section{Kalman Filtering}

In the standard least squares fit formalism for vertex reconstruction, all the tracks within an event are fitted to a single vertex in one single step. Due to this, it is often regarded as a global method. The dimensions of matrices and vectors in this formalism are proportional to the total number of measured tracks in the event, $N$. Because the required processing time for the inversion of a matrix is proportional to $N^{3}$, this method runs into trouble when trying to deal with events containing a high multiplicity of charged particles. Additionally, due to the global structure of this formalism, it is less flexible in handling different vertex hypotheses within a single event.

By contrast, the Kalman filter, which was originally designed to optimize dynamical 
systems, uses the information of different particle trajectories about the vertex consecutively one after another [24]. Because of this the Kalman filter is considered a local technique. Beginning with a start value for the vertex position, one compares this position with the information about the vertex from one track. By a correct weighting of the start value and the information from the track, one calculates a new estimate for the vertex position. This position is then compared with the information of the next particle track in the event producing another new estimate. This procedure is then repeated for each track one wants to fit to a single vertex, until one obtains the final vertex position. This technique is called filter.

\subsection{Summary}

The BABAR reconstruction package is the result of many people's work over the last few years. It reconstructs the many different event types at high speed $(\sim 10 \mathrm{~Hz})$ that are expected to occur in the interaction region of the BABAR experiment. It relies heavily on the quality of output from the various sub-detectors to function adequately. 


\section{Chapter 7}

\section{Multiple Mass Hypothesis Analysis}

The classification of particle species in the BABAR detector is of paramount importance if one wishes to observe $C P$ violation processes. Determining when it is acceptable to track unknown species as pions, as all five particle species, or as some subset of the five particle species in the detector plays a vital role in obtaining the best kinematic information for the events and hence plays a critical role in species classification.

\subsection{Methodology}

The first step in addressing the issue of multiple mass fits was to be able to read-in and analyze given events and then change the tracking particle hypothesis. The specific code needed was not in existence so a $\mathrm{C}++$ class was designed and constructed which was responsible for organizing the results of a given simulation into a form that would make it possible to examine. This code was inserted into the Beta framework and numerous computer simulations were carried out.

The computer simulations that were performed numbered four, one for each particle type (the pion was not addressed as it is the default choice in the fitting algorithm). For an individual mass hypothesis, approximately 10,000 Monte Carlo (MC) events were

considered, ranging in momenta from $50 \mathrm{MeV} / \mathrm{c}$ to $4.1 \mathrm{GeV} / \mathrm{c}$. The MC events included 
backgrounds and resolution, which fully simulated the detector response down to the electronics/readout and is based on GEANT [25]. Output simulated electron signals and is similar in format to the raw data read off the detector.

The track parameters chosen to examine the momentum dependence were:

1. $d$ (the distance to closest approach to the IP)

2. $\phi$

3. $z$

4. $p_{z}$

5. $p_{t}$

All but $d$ were examined at three locations in the detector,

1. Interaction Point (IP)

2. The entrance to the DCH (defined as the lowest wire hit)

3. The exit of the DCH (defined as the highest wire hit)

For the distance of closest approach to the IP, $d$, which is only defined at the IP, no other location was considered. Three locations were chosen because of the different aspects each one offers in testing the momentum dependence of the tracking results. The IP is important for vertexing and physics information, the entrance to the DCH is needed to help match tracks coming from the SVT, and the exit of the DCH is required to match tracks in the outer detectors (DIRC, EMC, IFR). 
The output from these track fits were stored in the form of HBOOK [26] files that were then loaded into Physics Analysis Workstation (PAW) [26] for further analysis. Once in PAW the results from the fits could be analyzed. A new parameter, $\Delta S$, was constructed which gauged the momentum dependence of a certain parameter. It was defined as,

$$
\Delta S(\text { parameter })=\frac{\text { parameter }(\text { WhatParticleIs })-\text { parameter }(\text { Pion })}{\text { parameterError }(\text { WhatParticleIs })}
$$

The units of $d$ and $z$ are in $\mathrm{cm}, \phi$ is in radians, $p_{z}$ and $p_{t}$ are in $\mathrm{GeV} / \mathrm{c}$ and $\Delta S$ has units of $\sigma$. In this way the result from tracking a particle as what it really is, for a given parameter, could be compared to the result of tracking this particle as a pion.

The parameter $\Delta S$ was plotted as a function of Monte Carlo momentum to observe its momentum dependency. To facilitate statistical analysis, the full momentum range was sub-divided into $100 \mathrm{MeV} / \mathrm{c}$ partitions. The $\Delta S$ parameter was then examined in each of these momentum "slices." The momentum cutoff was defined to be the maximum momentum "slice" at which $\Delta S$ failed to be less than $0.1 \sigma$ at a $95 \%$ Confidence Level (CL). This investigation was repeated for each parameter at each location for all four computer simulations.

\subsection{Electron Results}

The momentum dependence of $\Delta S$ for the five chosen parameters at the IP is shown in figures 7.1 and 7.2. The various electron cutoff values are summarized in table 7.1. After looking through the table, the limiting case was found to be for the parameter $p_{t}$ at the IP. This momentum "slice" is shown in figure 7.3. 


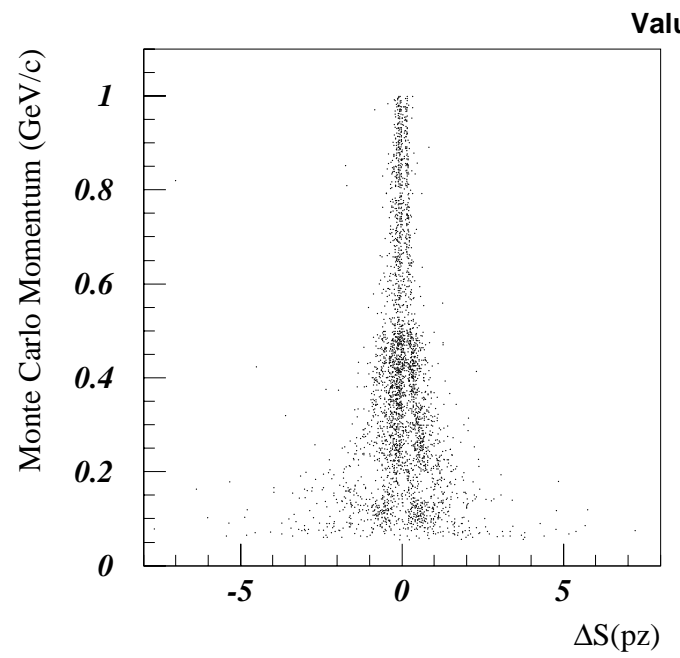

Values at the IP
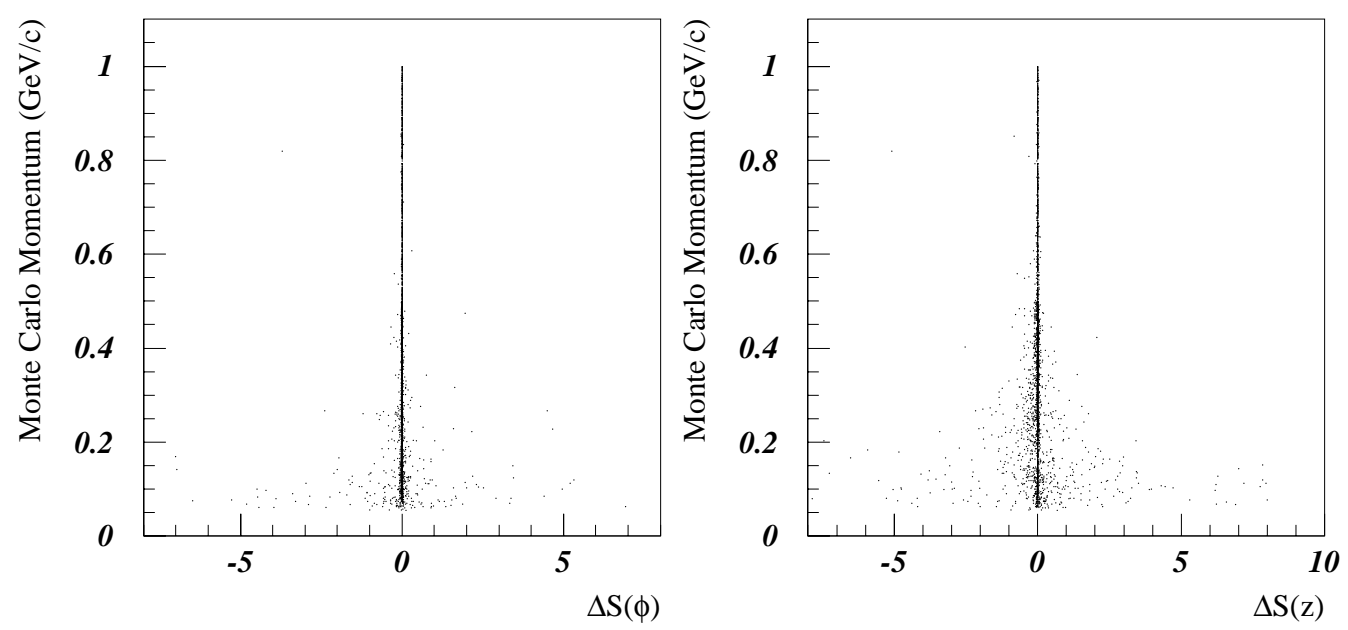

Figure 7.1: $\Delta S$ vs. Monte Carlo Momentum for $p_{z}, d, \phi$, and $z$ for an Electron. 


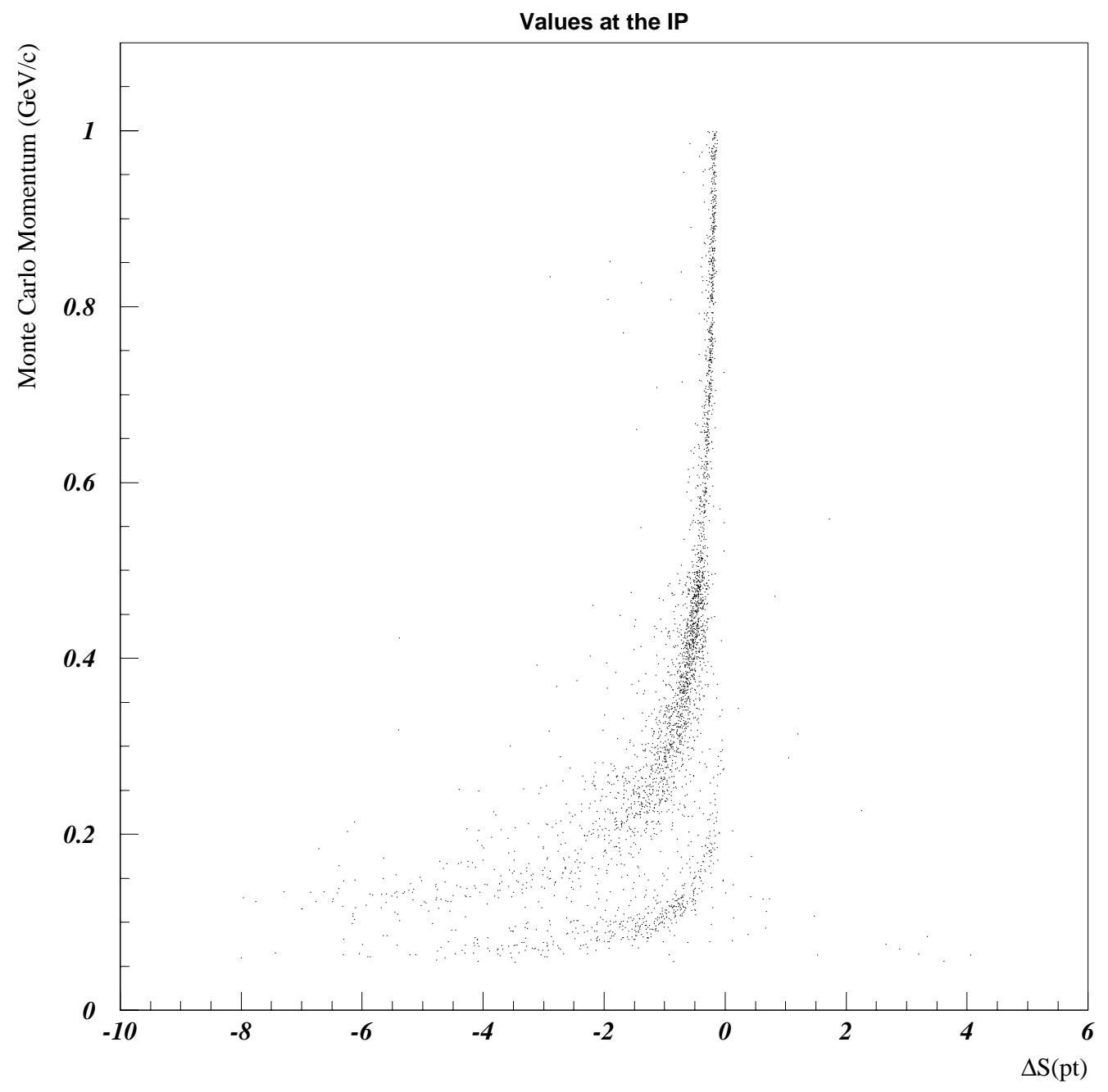

Figure 7.2: $\Delta S\left(p_{t}\right)$ vs. Monte Carlo Momentum for an Electron. 


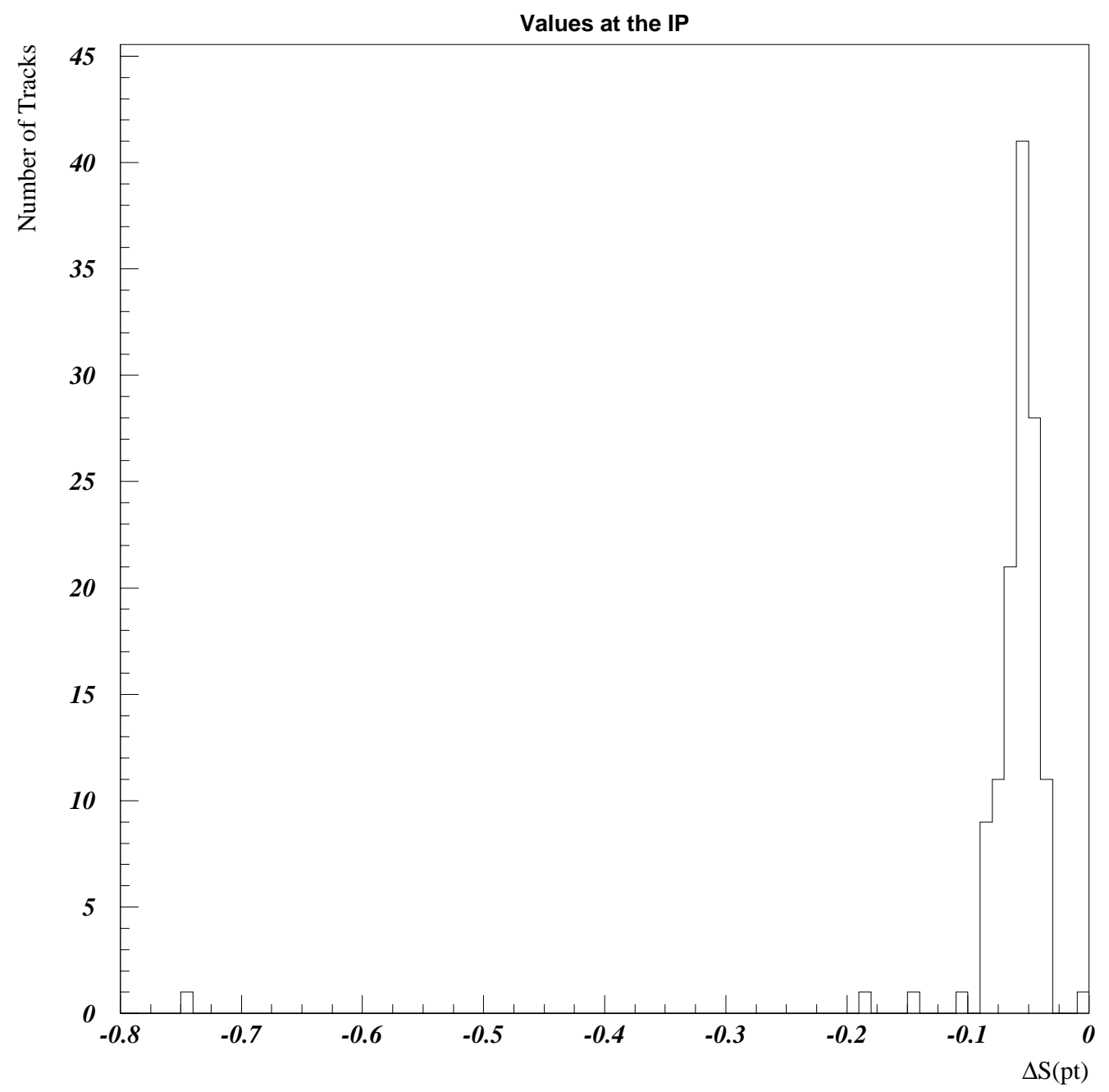

Figure 7.3: Electron $\Delta S\left(p_{t}\right)$ distribution for $2.1 \leq p \leq 2.2 \mathrm{GeV} / \mathrm{c}$ 


\begin{tabular}{|c|c|c|c|}
\hline & IP & DCH ENTER & DCH EXIT \\
\hline$d$ & $0.4(566)$ & - & - \\
\hline$\phi$ & $0.3(506)$ & $0.5(151)$ & $0.7(138)$ \\
\hline$z$ & $0.5(149)$ & $0.3(499)$ & $0.5(137)$ \\
\hline$p_{z}$ & $2.1(126)$ & $0.3(499)$ & $0.5(137)$ \\
\hline$p_{t}$ & $2.1(126)$ & $0.3(499)$ & $0.5(138)$ \\
\hline
\end{tabular}

Table 7.1: Electron Momentum Cutoffs $(\mathrm{GeV} / \mathrm{c})$ for $\Delta S<0.1 \sigma$ at a $95 \% \mathrm{CL}$. The numbers in brackets indicate the number of tracks in the $100 \mathrm{MeV}$ "slice" starting at the indicated momentum.

\begin{tabular}{|c|c|c|c|}
\hline & IP & DCH ENTER & DCH EXIT \\
\hline$d$ & $0.4(466)$ & - & - \\
\hline$\phi$ & $0.2(525)$ & $0.5(160)$ & $0.5(145)$ \\
\hline$z$ & $0.5(169)$ & $0.5(160)$ & $0.5(145)$ \\
\hline$p_{z}$ & $0.4(466)$ & $0.2(444)$ & $0.4(370)$ \\
\hline$p_{t}$ & $0.5(169)$ & $0.2(443)$ & $0.4(372)$ \\
\hline
\end{tabular}

Table 7.2: Muon Momentum Cutoffs $(\mathrm{GeV} / \mathrm{c})$ for $\Delta S<0.1 \sigma$ at a $95 \% \mathrm{CL}$. The numbers in brackets indicate the number of tracks in the $100 \mathrm{MeV}$ "slice" starting at the indicated momentum.

\subsection{Muon Results}

The momentum dependence of $\Delta S$ for the five chosen parameters at the IP is shown in figures 7.4 and 7.5. The various muon cutoff values are summarized in table 7.2. After looking through the table, the limiting case was found to be for the parameter $p_{t}$ at the IP. This momentum "slice" is shown in figure 7.6. 


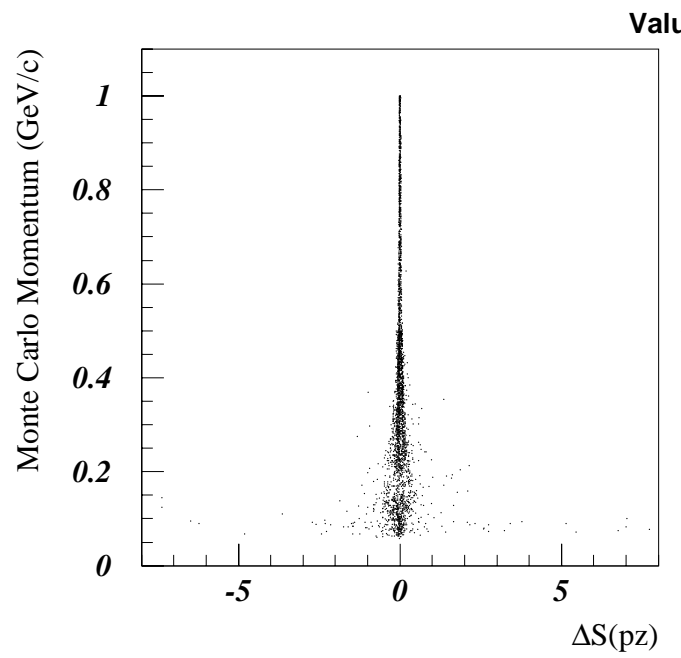

Values at the IP
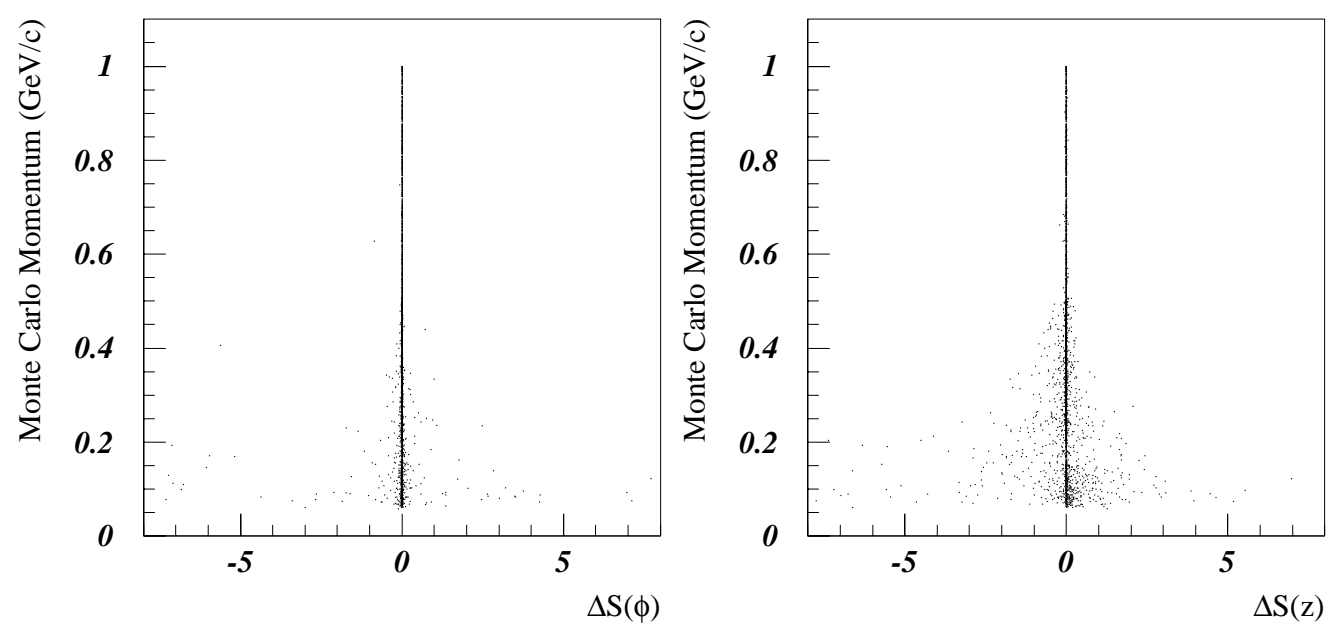

Figure 7.4: $\Delta S$ vs. Monte Carlo Momentum for $p_{z}, d, \phi$, and $z$ for a Muon. 


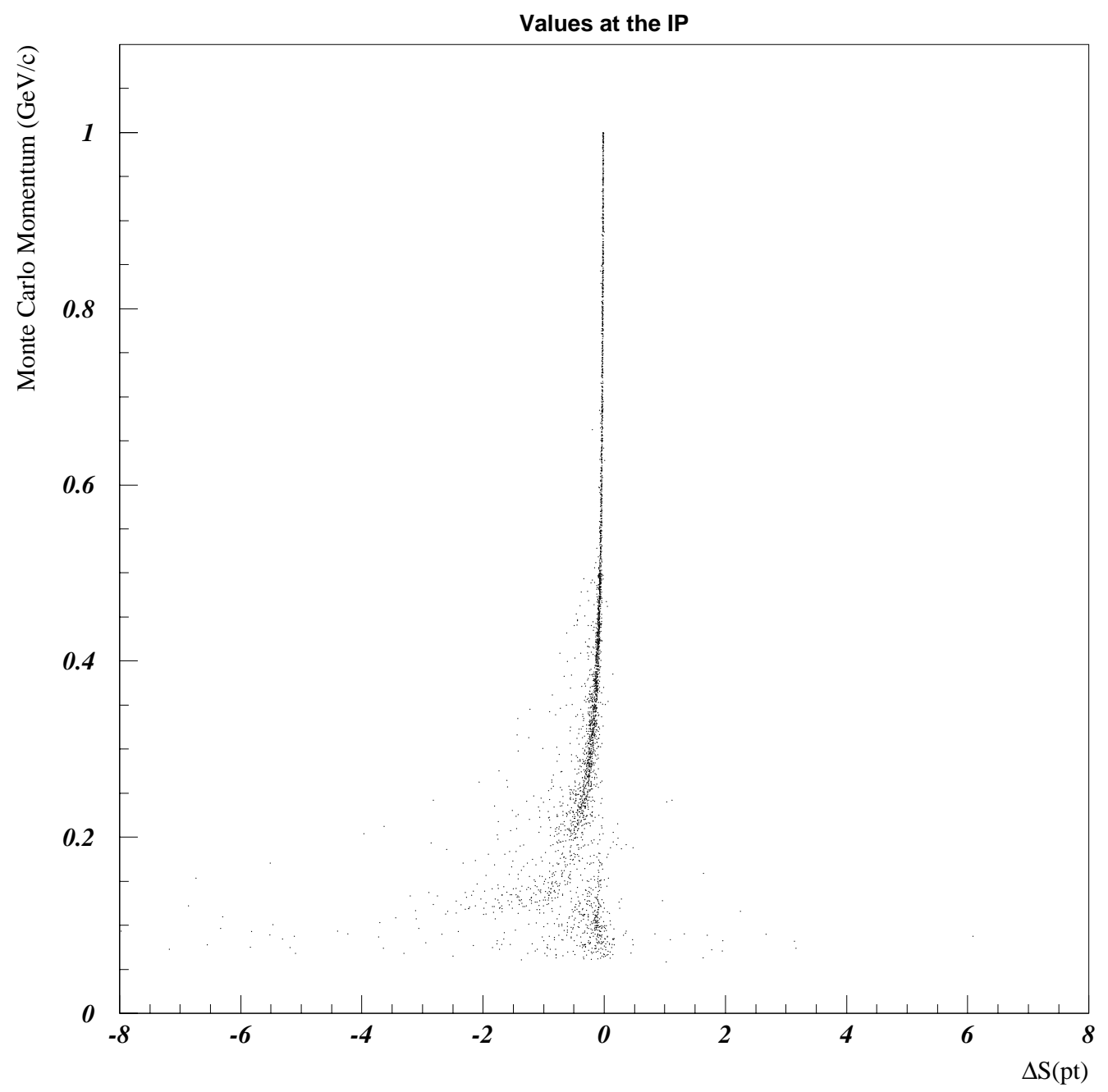

Figure 7.5: $\Delta S\left(p_{t}\right)$ vs. Monte Carlo Momentum for a Muon. 


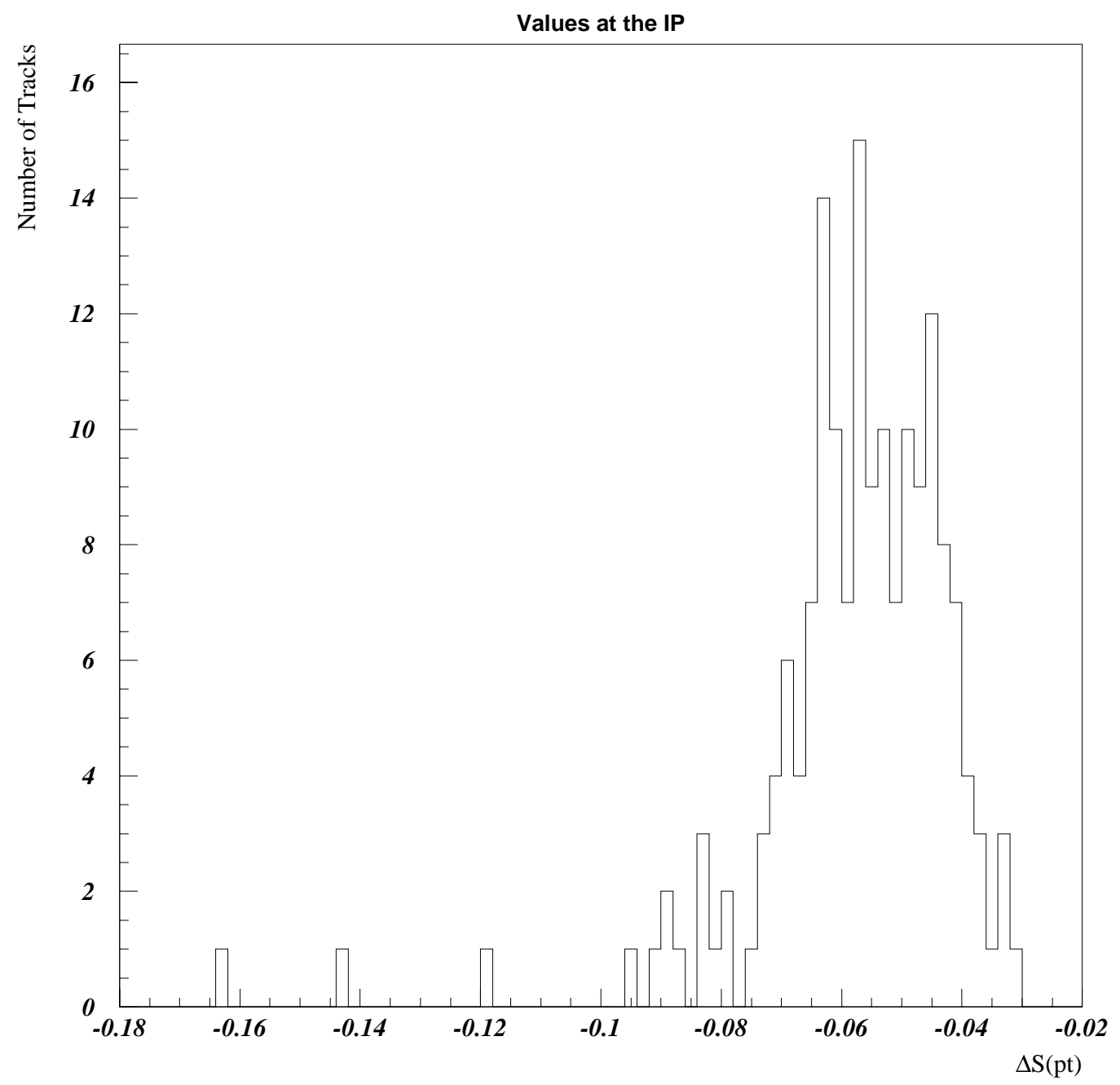

Figure 7.6: Muon $\Delta S\left(p_{t}\right)$ distribution for $0.5 \leq p \leq 0.6 \mathrm{GeV} / \mathrm{c}$ 


\begin{tabular}{|c|c|c|c|}
\hline & IP & DCH ENTER & DCH EXIT \\
\hline$d$ & $1.3(145)$ & - & - \\
\hline$\phi$ & $0.4(406)$ & $1.2(107)$ & $1.1(95)$ \\
\hline$z$ & $1.3(145)$ & $1.5(124)$ & $1.7(112)$ \\
\hline$p_{z}$ & $2.0(97)$ & $1.4(146)$ & $1.2(106)$ \\
\hline$p_{t}$ & $1.9(131)$ & $1.5(124)$ & $1.2(106)$ \\
\hline
\end{tabular}

Table 7.3: Kaon Momentum Cutoffs $(\mathrm{GeV} / \mathrm{c})$ for $\Delta S<0.1 \sigma$ at a $95 \% \mathrm{CL}$. The numbers in brackets indicate the number of tracks in the $100 \mathrm{MeV}$ "slice" starting at the indicated momentum.

\subsection{Kaon Results}

The momentum dependence of $\Delta S$ for the five chosen parameters at the IP is shown in figures 7.7 and 7.8. The various kaon cutoff values are summarized in table 7.3. After looking through the table, the limiting case was found to be for the parameter $p_{z}$ at the IP. This momentum "slice" is shown in figure 7.9 .

\subsection{Proton Results}

The momentum dependence of $\Delta S$ for the five chosen parameters at the IP is shown in figures 7.10 and 7.11. The various proton cutoff values are summarized in table 7.4. After looking through the table, the limiting case was found to be for the parameter $p_{t}$ at the IP. This momentum "slice" is shown in figure 7.12.

\subsection{The Effect of Changing the Sigma Value}

Due to the time constraints placed on the tracking/reconstruction software and the extra time required to refit tracks using multiple mass hypotheses, additional analysis 


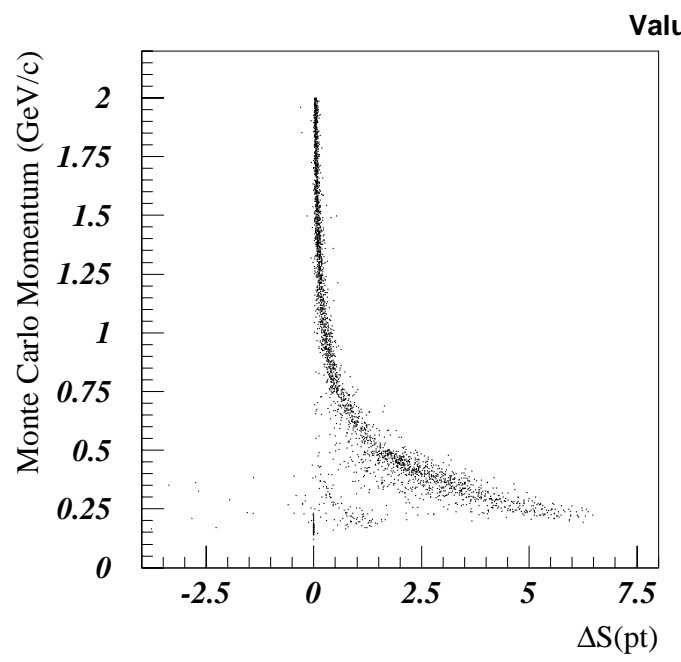

Values at the IP
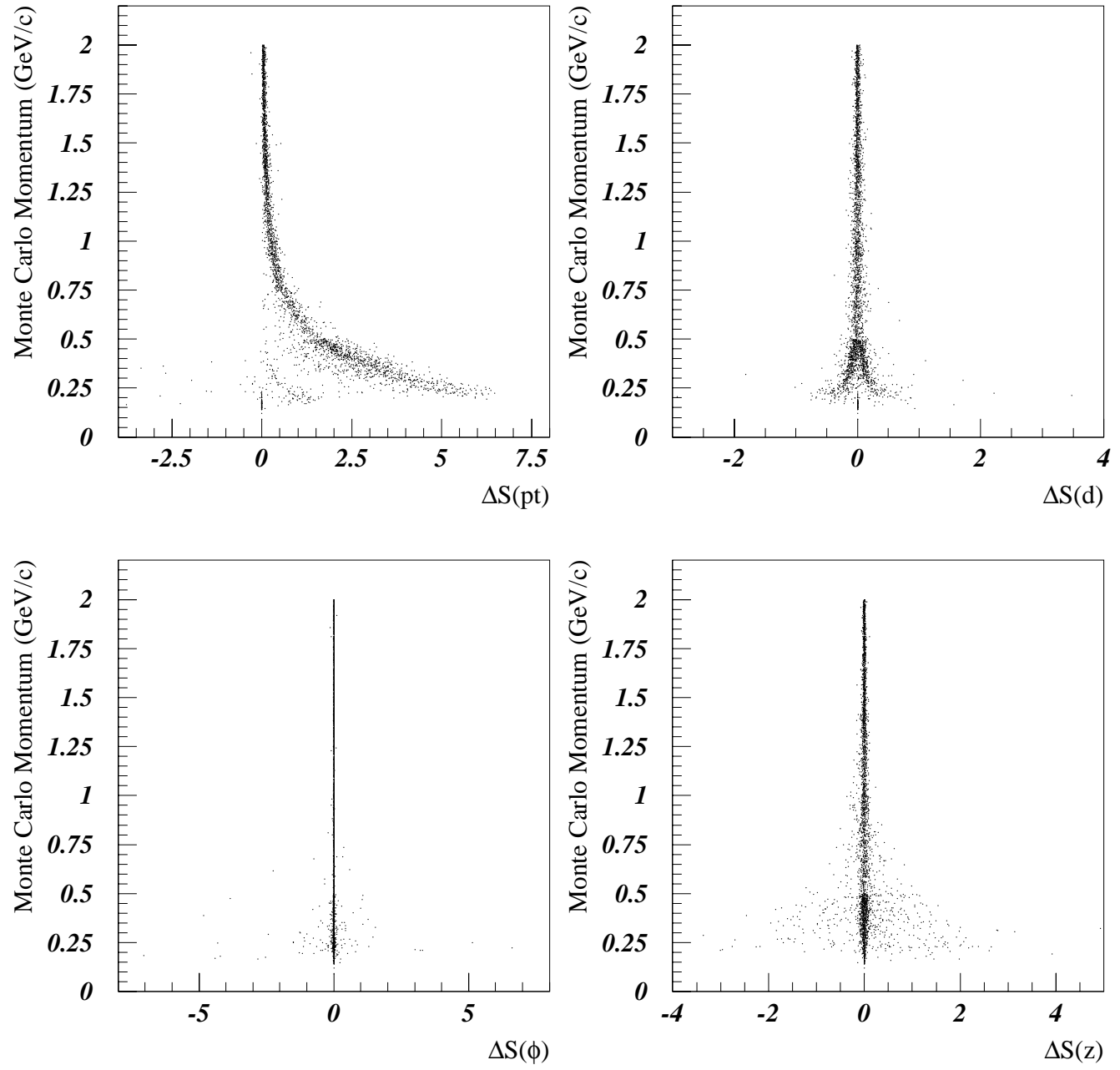

Figure 7.7: $\Delta S$ vs. Monte Carlo Momentum for $p_{t}, d, \phi$, and $z$ for a Kaon. 


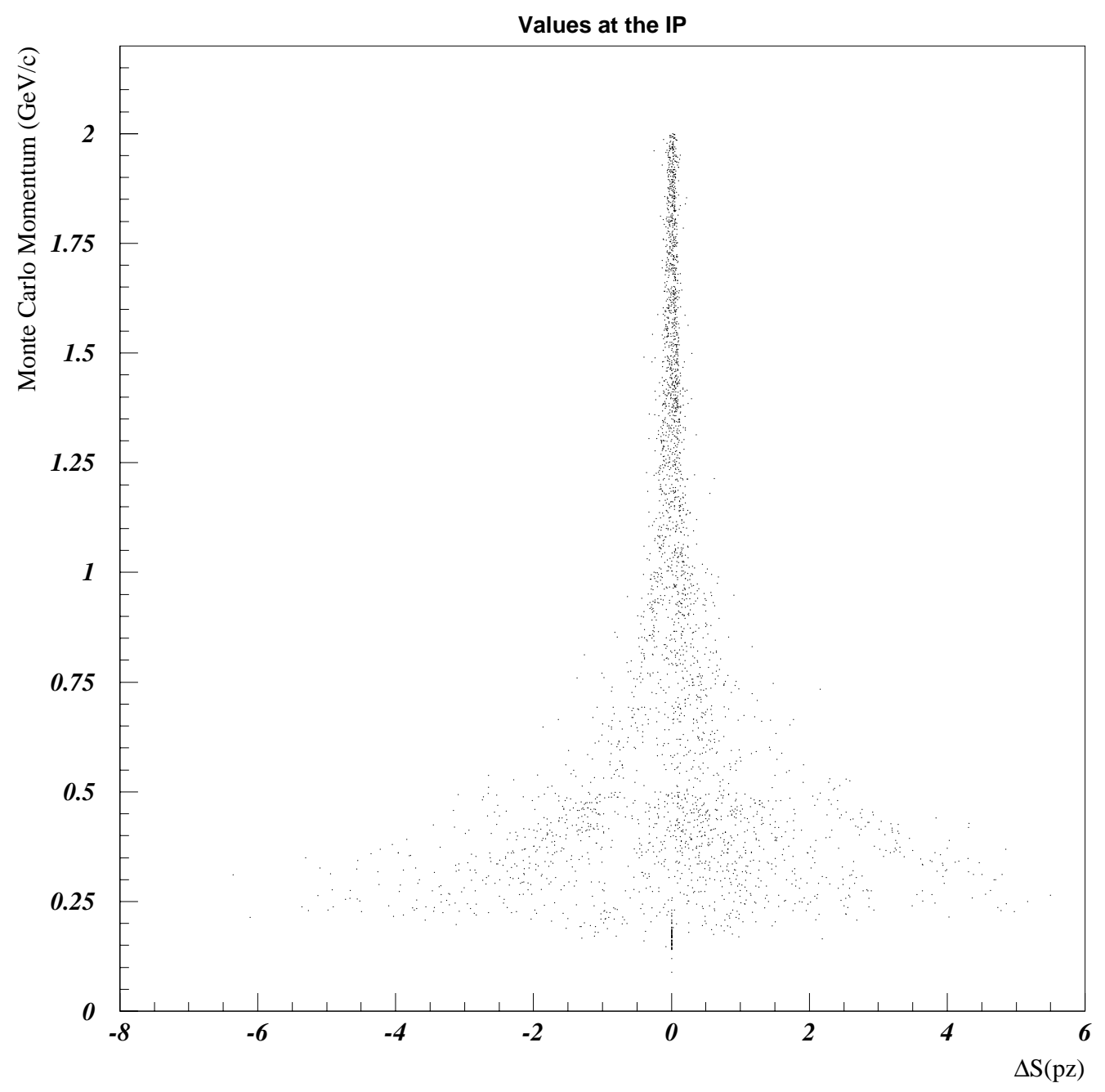

Figure 7.8: $\Delta S\left(p_{z}\right)$ vs. Monte Carlo Momentum for a Kaon 


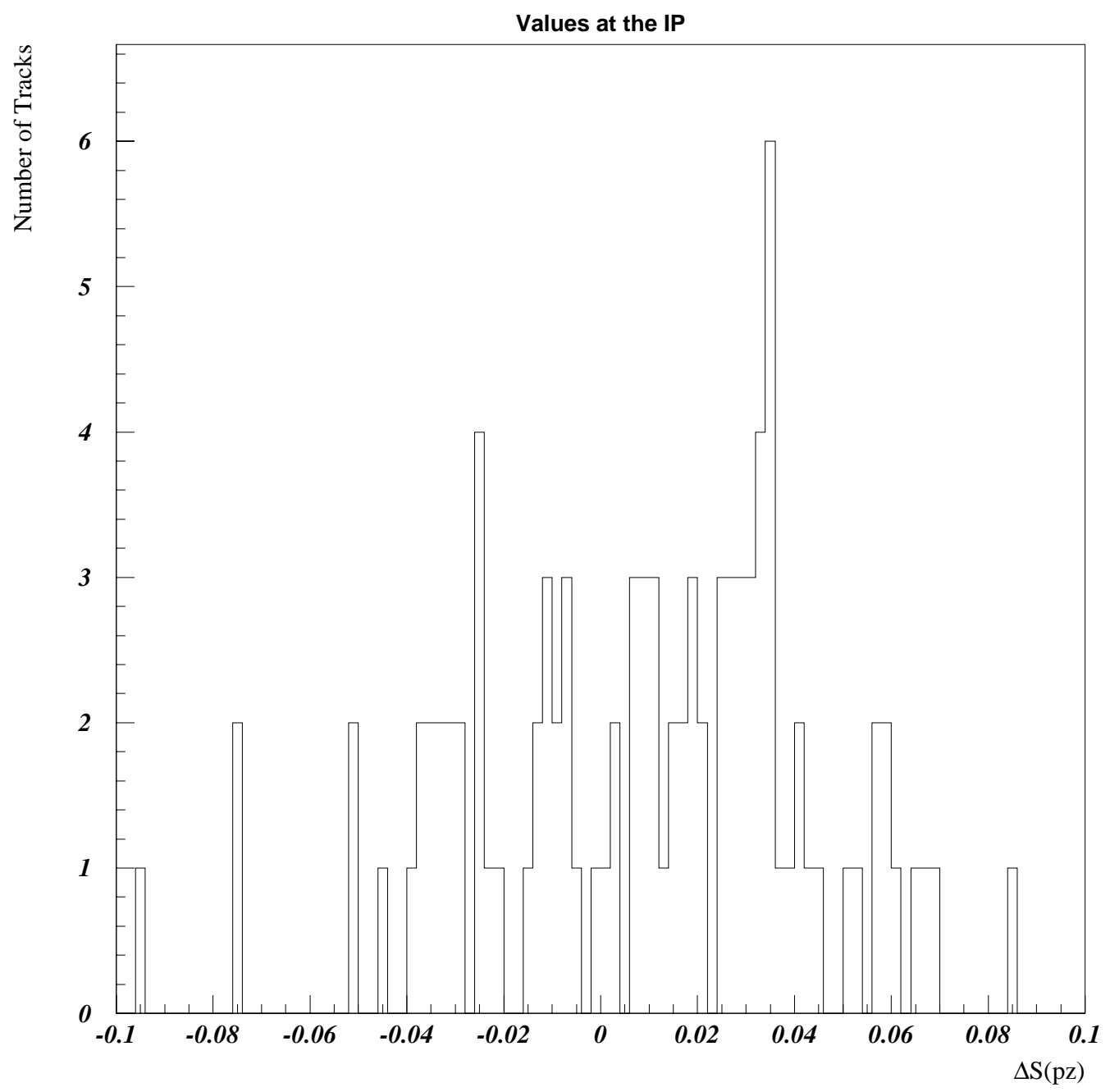

Figure 7.9: Kaon $\Delta S\left(p_{z}\right)$ distribution for $2.0 \leq p \leq 2.1 \mathrm{GeV} / \mathrm{c}$ 

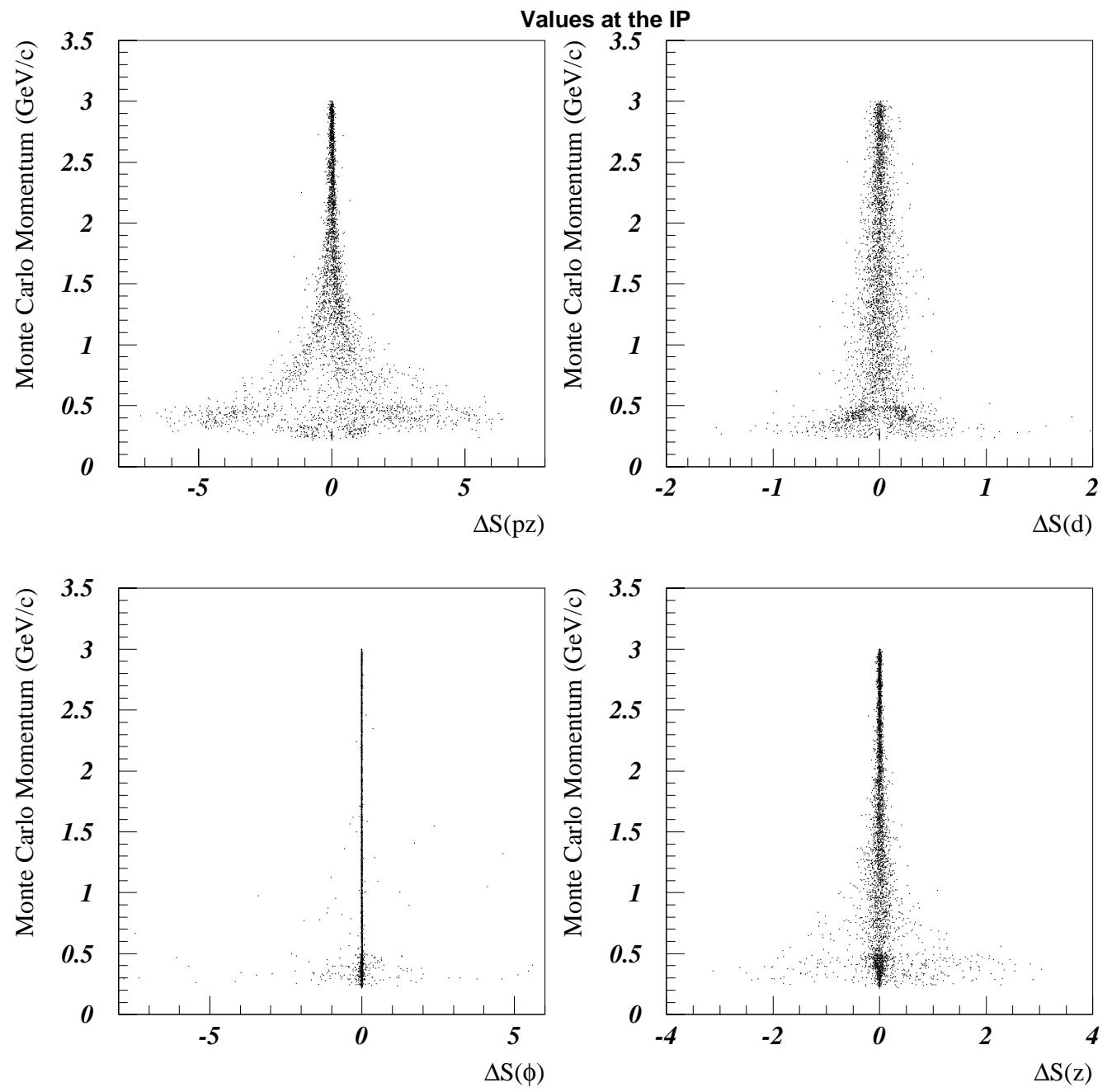

Figure 7.10: $\Delta S$ vs. Monte Carlo Momentum for $p_{z}, d, \phi$, and $z$ for a Proton 


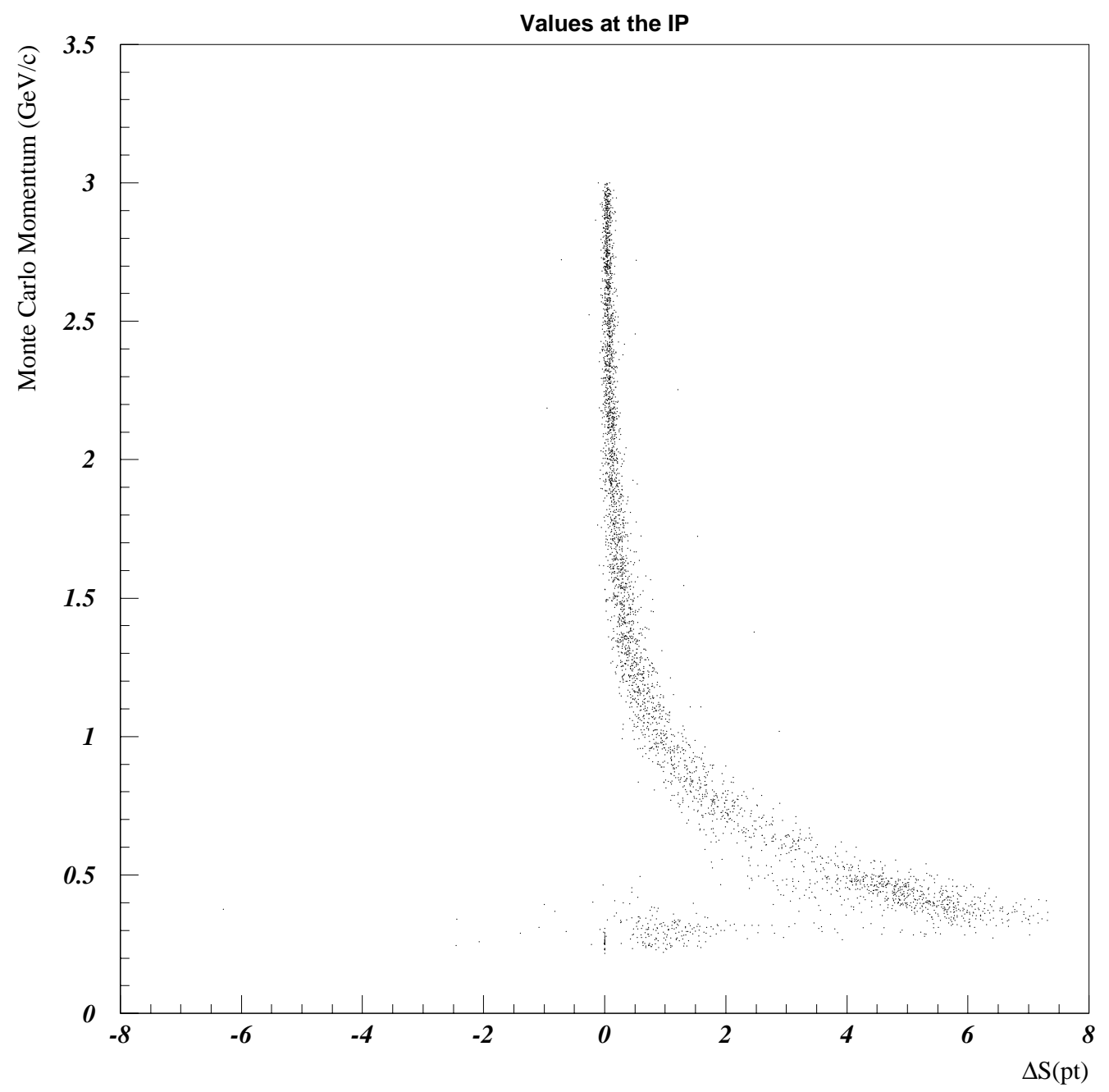

Figure 7.11: $\Delta S\left(p_{t}\right)$ vs. Monte Carlo Momentum for a Proton 


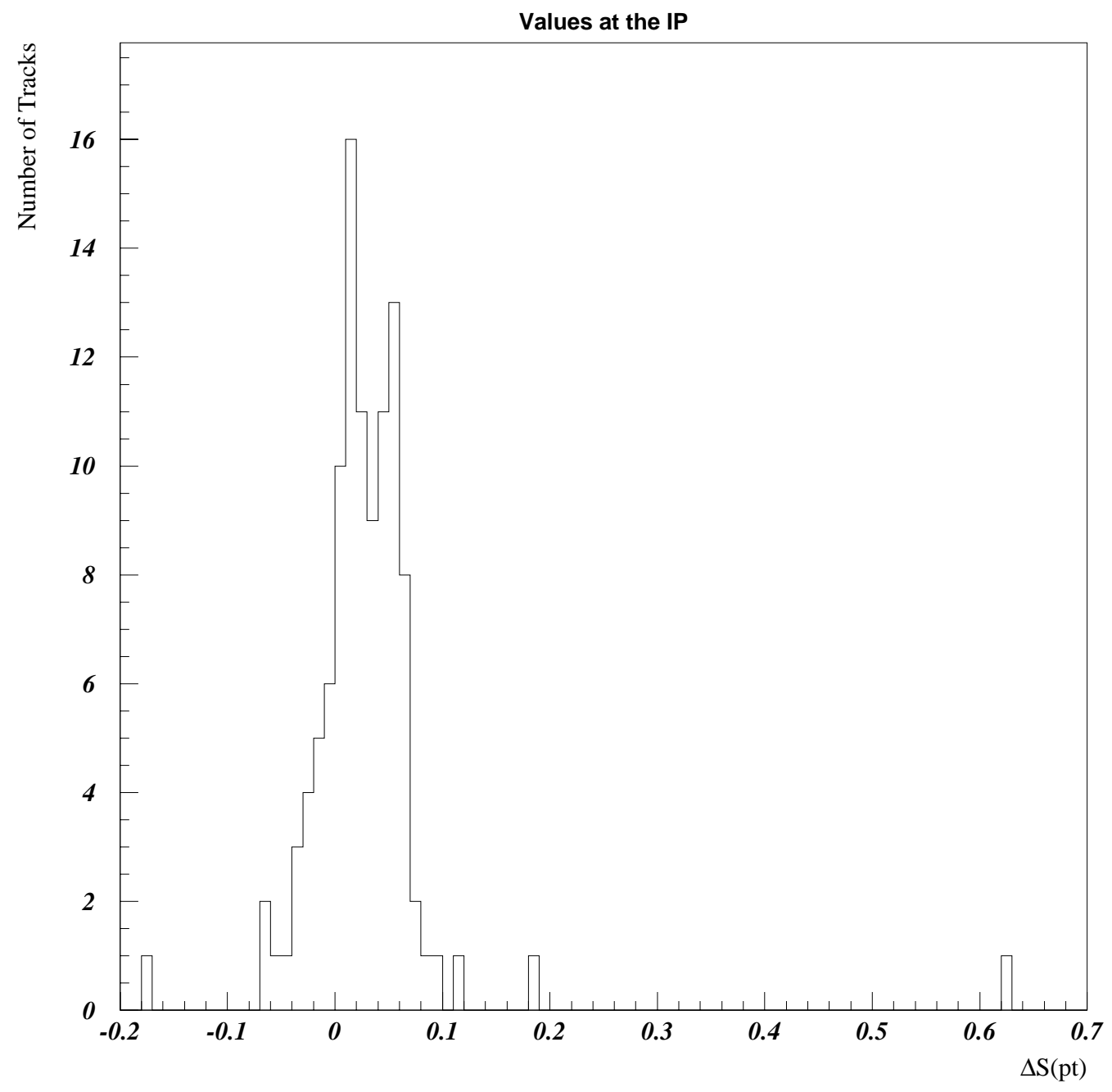

Figure 7.12: Proton $\Delta S\left(p_{t}\right)$ distribution for $3.1 \leq p \leq 3.2 \mathrm{GeV} / \mathrm{c}$ 


\begin{tabular}{|c|c|c|c|}
\hline & IP & DCH ENTER & DCH EXIT \\
\hline$d$ & $2.7(126)$ & - & - \\
\hline$\phi$ & $0.5(97)$ & $2.0(93)$ & $2.0(91)$ \\
\hline$z$ & $2.0(94)$ & $2.2(108)$ & $2.9(103)$ \\
\hline$p_{z}$ & $2.9(106)$ & $2.4(110)$ & $2.4(107)$ \\
\hline$p_{t}$ & $3.1(108)$ & $2.7(125)$ & $2.7(122)$ \\
\hline
\end{tabular}

Table 7.4: Proton Momentum Cutoffs $(\mathrm{GeV} / \mathrm{c})$ for $\Delta S<0.1 \sigma$ at a $95 \% \mathrm{CL}$. The numbers in brackets indicate the number of tracks in the $100 \mathrm{MeV}$ "slice" starting at the indicated momentum.

Effect of Changing the Sigma Value on Cutoff Momentum

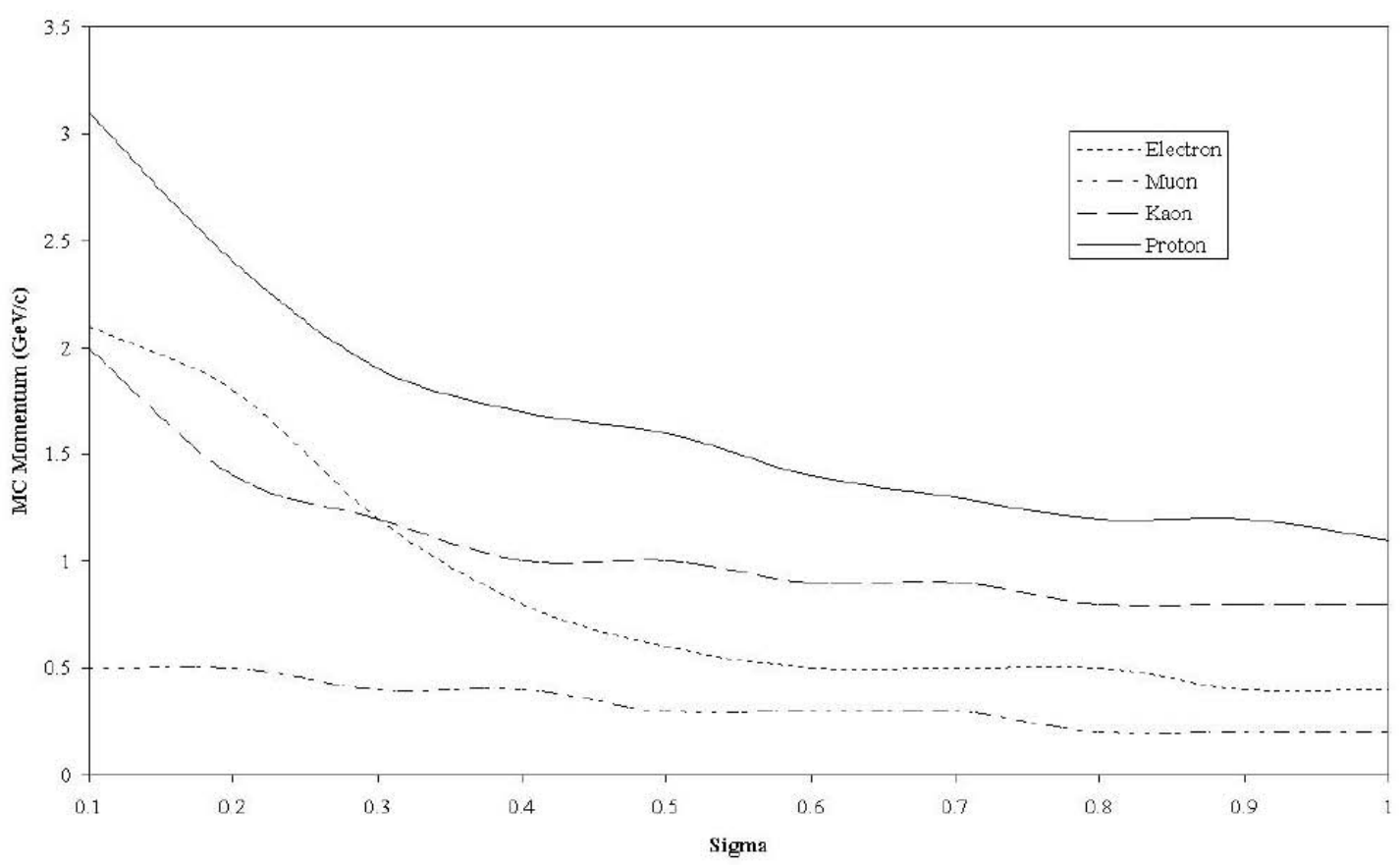

Figure 7.13: The Effect of Changing the Sigma Value on Cutoff Momentum. 


\begin{tabular}{|c|c|c|c|c|}
\hline$\Delta S<$ & Electron & Muon & Kaon & Proton \\
\hline $0.1 \sigma$ & $2.1(126)$ & $0.5(169)$ & $2.0(131)$ & $3.1(108)$ \\
\hline $0.2 \sigma$ & $1.8(146)$ & $0.5(169)$ & $1.4(146)$ & $2.4(107)$ \\
\hline $0.3 \sigma$ & $1.2(145)$ & $0.4(466)$ & $1.2(107)$ & $1.9(107)$ \\
\hline $0.4 \sigma$ & $0.8(157)$ & $0.4(466)$ & $1.0(105)$ & $1.7(113)$ \\
\hline $0.5 \sigma$ & $0.6(144)$ & $0.3(497)$ & $1.0(152)$ & $1.6(109)$ \\
\hline $0.6 \sigma$ & $0.5(150)$ & $0.3(497)$ & $0.9(152)$ & $1.4(118)$ \\
\hline $0.7 \sigma$ & $0.5(150)$ & $0.3(497)$ & $0.9(118)$ & $1.3(128)$ \\
\hline $0.8 \sigma$ & $0.5(150)$ & $0.2(498)$ & $0.8(118)$ & $1.2(135)$ \\
\hline $0.9 \sigma$ & $0.4(564)$ & $0.2(498)$ & $0.8(117)$ & $1.2(135)$ \\
\hline $1.0 \sigma$ & $0.4(564)$ & $0.2(498)$ & $0.8(117)$ & $1.1(116)$ \\
\hline
\end{tabular}

Table 7.5: Momentum Cutoffs $(\mathrm{GeV} / \mathrm{c})$ for Various Values of $\Delta S$ at a $95 \% \mathrm{CL}$. The numbers in brackets indicate the number of tracks in the $100 \mathrm{MeV}$ range starting at the indicated momentum.

was done using various acceptable values of $\Delta S$. The tight constraint of $\Delta S<0.1 \sigma$ at 95\% CL was relaxed in $0.1 \sigma$ steps and its effect on the momentum cutoff values were tallied (table 7.5). This effect is also shown graphically in figure 7.13.

\subsection{Recommendation}

Instead of using an "all or nothing" approach to tracking the unknown species in the detector as pions or as all five particle species, a cascading cutoff is recommended. Due to the wide momentum range between the various cutoffs for the four particle species it would be beneficial to only turn on fitting of each particle species as it is warranted. For the original requirement that the parameter $\Delta S$ be less than $0.1 \sigma$ at a $95 \%$ CL the momentum cutoffs for the respective particle species are:

$$
\begin{array}{r}
\text { Electron } 2.1 \mathrm{GeV} / \mathrm{c} \\
\text { Muon } 0.5 \mathrm{GeV} / \mathrm{c}
\end{array}
$$




\section{Kaon $2.0 \mathrm{GeV} / \mathrm{c}$ \\ Proton $3.1 \mathrm{GeV} / \mathrm{c}$}

(eg. For momenta greater than $3.1 \mathrm{GeV} / \mathrm{c}$, fit track as a pion only. For momenta greater than $2.1 \mathrm{GeV} / \mathrm{c}$, fit track as a pion and proton. For momenta greater than $2.0 \mathrm{GeV} / \mathrm{c}$, fit track as a pion, proton, and electron. For momenta greater than $0.5 \mathrm{GeV} / \mathrm{c}$, fit track as a pion, proton, electron, and kaon. For values of momenta below $0.5 \mathrm{GeV} / \mathrm{c}$, fit track as all 5 particle species.) These values, along with the cascading condition, are currently being implemented in version 7.11 .1 of the BABAR reconstruction software. Currently work is being done to optimize performance vs. CPU time using the momentum cutoffs given in table 7.5 . 


\section{Chapter 8}

\section{Conclusion}

Deciding when it is necessary to track particles of unknown species in the BABAR detector as pions or as all five particle species is crucial for both computational time constraints and the accuracy of track fit results. Naively, one could choose to always track particles in the detector as all five particle species. This dramatically increases the processing time required for each track and is highly inefficient. This thesis deals with an analysis of Monte Carlo events in an effort to quantify the momentum cutoffs of the five particle species in the detector. The results of this analysis will aid in BABAR's goal of quickly and cleanly observing $C P$ violation in the neutral $B$ system.

\subsection{Summary}

The code to implement the cascading condition algorithm is currently installed and in use in the BABAR reconstruction software version 7.11.1. The values for the momentum cut-offs for the four particle species are also included in the above code. 


\section{Bibliography}

[1] A.D. Sakharov Soviet Physics Usp 34, 5 (1991).

[2] J.H. Christenson et al. Physical Review Letters 13, 138 (1964).

[3] M. Kobayashi and T. Maskawa, "CP Violation in the Renormalizable Theory of Weak Interaction",Progress Theoretical Physics, 49, 652 (1973).

[4] G. Farrar, "CP Violation and the Baryonic Asymmetry of the Universe", Nuclear Physics B, Proceedings, Supplements, 43, 312 (1995).

[5] Y. Nir and H. Quinn, The BABAr Physics Book, SLAC Report 504, October 1998.

[6] M. Gell-Mann and A. Païs, "Behavior of Neutral Particles Under Charge Conjugation", Physical Review Letters, 97, 1387 (1955).

[7] Particle Data Group, C. Caso et al., The European Physical Journal C3, 1 (1998).

[8] J.H. Christenson, J.W. Cronin, V.L. Fitch and R. Turlay, "Evidence for the $2 \pi$ decay of the $K_{2}^{0}$ meson", Physical Review Letters, 13, 138 (1964).

[9] P. Oddone, in Proceedings of the UCLA Workshop: Linear Collider B $\bar{B}$ Factory Conceptual Design, edited by D. Stork, World Scientific (1987), p. 243.

[10] "Technical Design Report," (BABAR Collaboration), SLAC-REP-372 (1995).

[11] A. Snyder, "The Effect of Vertex Cuts on CP Reach," BaBar Note \#177 (1994).

[12] G. Charpak, "Applications of proportional chambers and drift chambers in highenergy physics and other fields," Nature, 270, (1977).

[13] W.R. Leo, Techniques for Nuclear and Particle Physics Experiments, SpringerVerlag, 1992.

[14] R.C. Weast and S.M. Selby, Eds., CRC Handbook of Chemistry and Physics, fortyeightth edition, The Chemical Rubber Company, 1967.

[15] J. Heise, "Studies of Helium-Based Gas Mixtures Using a Small Cell Drift Chamber", UBC M.Sc. Thesis, 1996. 
[16] P. Rice-Evans, Spark, Streamer, Proportional and Drift Chambers, The Richelieu Press, London, 1974.

[17] S. Uno, et al., "Study of a drift chamber filled with a helium-ethane mixture", Nuclear Instruments and Methods in Physics Research, A330, 55 (1993).

[18] J.M. McKenzie, " Development of the Semiconductor Radiation Detector", Nuclear Instruments and Methods in Physics Research, 162, 49 (1979).

[19] J. Millman and C.C. Halkias, Integrated Electronics: Analog and Digital Circuits and Systems, McGraw-Hill Book Company, 1972.

[20] B. Hyams, et al., "A Silicon Counter Telescope to Study Shortlived Particles in High-Energy Hadronic Interactions", Nuclear Instruments and Methods in Physics Research, 205, 99 (1983).

[21] D. Barbieri et al., "Silicon Sensors for the BABAR Vertex Tracker," submitted to Nuovo Cimento.

[22] E.D. Frank et al., "Architecture of the BABAR Reconstruction System," to be published in Computer Physics Communications.

[23] B. Jacobsen, "Beta: A High-level Toolkit for BABAr Physics Analysis," submitted to Computer Physics Communications.

[24] R. Luchsinger and C. Grab, "Vertex reconstruction by means of the method of Kalman filtering," Computer Physics Communications, 76, 1993.

[25] R. Brun et al., "GEANT: Simulation Program for Particle Physics Experiments. User Guide and Reference Manual", CERN-DD/78/2 Rev., July 1978.

[26] R. Brun et al., "Physics Analysis Workstation", CERNLIB, Q121, 1989. 